\title{
A Crucial Role for Primary Cilia in Cortical Morphogenesis
}

\author{
Marc A. Willaredt, ${ }^{1,2}$ Kerstin Hasenpusch-Theil, ${ }^{6}$ Humphrey A. R. Gardner, ${ }^{7}$ Igor Kitanovic, ${ }^{4}$ \\ Vera C. Hirschfeld-Warneken, ${ }^{3,5}$ Christian P. Gojak, ${ }^{3,5}$ Karin Gorgas, ${ }^{2}$ C. Lulu Bradford, ${ }^{1}$ Joachim Spatz, ${ }^{3,5}$ Stefan Wölfl, \\ Thomas Theil, ${ }^{6}$ and Kerry L. Tucker ${ }^{1,2}$ \\ ${ }^{1}$ Interdisciplinary Center for Neurosciences, ${ }^{2}$ Department of Anatomy, ${ }^{3}$ Department of Biophysical Chemistry, Institute for Physical Chemistry, and \\ ${ }^{4}$ Institute of Pharmacy and Molecular Biotechnology, University of Heidelberg, 69120 Heidelberg, Germany, ${ }^{5}$ Max Planck Institute for Metals Research, \\ 70569 Stuttgart, Germany, ${ }^{6}$ Centres for Neuroscience Research and Integrative Physiology, University of Edinburgh, Edinburgh EH8 9XD, United Kingdom, \\ and ${ }^{7}$ Novartis Institutes for BioMedical Research, Cambridge, Massachusetts 02139
}

Primary cilia are important sites of signal transduction involved in a wide range of developmental and postnatal functions. Proteolytic processing of the transcription factor Gli3, for example, occurs in primary cilia, and defects in intraflagellar transport (IFT), which is crucial for the maintenance of primary cilia, can lead to severe developmental defects and diseases. Here we report an essential role of primary cilia in forebrain development. Uncovered by $N$-ethyl- $N$-nitrosourea-mutagenesis, cobblestone is a hypomorphic allele of the IFT gene Ift 88 , in which Ift $88 \mathrm{mRNA}$ and protein levels are reduced by $70-80 \%$. cobblestone mutants are distinguished by subpial heterotopias in the forebrain. Mutants show both severe defects in the formation of dorsomedial telencephalic structures, such as the choroid plexus, cortical hem and hippocampus, and also a relaxation of both dorsal-ventral and rostral-caudal compartmental boundaries. These defects phenocopy many of the abnormalities seen in the Gli3 mutant forebrain, and we show that Gli3 proteolytic processing is reduced, leading to an accumulation of the full-length activator isoform. In addition, we observe an upregulation of canonical Wnt signaling in the neocortex and in the caudal forebrain. Interestingly, the ultrastructure and morphology of ventricular cilia in the cobblestone mutants remains intact. Together, these results indicate a critical role for ciliary function in the developing forebrain.

Key words: primary cilia; cortex; Gli3; Wnt; Ift88; intraflagellar transport

\section{Introduction}

Cilia are microtubule-based organelles protruding from the surface of most cells in the vertebrate body, which are found across the phylogenetic spectrum (Davis et al., 2006). Primary cilia, which lack an innermost microtubule doublet, have recently been implicated in a wide variety of developmental functions, including left-right asymmetry and limb, kidney, pancreas, and skeleton formation (Bisgrove and Yost, 2006). In the developing nervous system, mutants of genes involved in ciliary formation and maintenance display defects in neural tube closure, flexure and neuronal differentiation (Murcia et al., 2000; Huangfu et al., 2003; Huangfu and Anderson, 2005; Liu et al., 2005; May et al., 2005; Houde et al., 2006; Caspary et al., 2007), and perinatal hippocampal (Han et al., 2008) and cerebellar (Chizhikov et al.,

Received May 4, 2008; revised Aug. 8, 2008; accepted 0ct. 15, 2008.

This work was supported by the German Research Foundation (K.L.T., Deutsche Forschungsgemeinschaft, Sonderforschungsbereich 488), the University of Heidelberg (K.L.T.), and the Wellcome Trust (T.T.). We thank YvesAlain Barde for supporting the ENU screen, Joachim Kirsch for generous scientific support, Andrew Peterson for ENU mutagenesis advice, Bradley Yoder (University of Albama at Birmingham Recessive Polycystic Kidney Disease Research and Translational Core (enter 5 P30 DK074038-02) and Venus Roper for mouse lines Ift88 $8^{\text {tm } 1.1 B k y}$ and Ift88 ${ }^{\Delta 2-3 \beta g a l}$, Ulrich Rüther for the $X t^{\prime}$ line, Joseph Besharse for anti-Ift88 antibodies, James Briscoe for the Shh reporter plasmid, David Wilkinson, Alexandra Joyner, John Mason, Ulrich Rüther, and Matthias Treier for in situ probes, Ho Li for in silico SNP primer evaluation, Vassiliki Fotaki for Gli3 Western advice, Sophia Bardehle for setting up real-time RT-PCR, David Lau for help with luciferase assays, and Angela Scuderi, Bernice Pawletta, Xiao Shen, Xiao-Rui Sun, Valentin Evsyukov, and Christina Spassova for excellent technical assistance.

Correspondence should be addressed to Dr. Kerry L. Tucker, University of Heidelberg, Im Neuenheimer Feld 307, 69120 Heidelberg, Germany. E-mail: Kerry.Tucker@urz.uni-hd.de.

DOI:10.1523/JNEUROSCI.2084-08.2008

Copyright $\odot 2008$ Society for Neuroscience $\quad 0270-6474 / 08 / 2812887-14 \$ 15.00 / 0$
2007; Spassky et al., 2008) development. Cilia have been reported to be present in neurons throughout the nervous system (Fuchs and Schwark, 2004) and also in proliferating neuroepithelia that extend cilia into the ventricular space (Nagele and Lee, 1979; Cohen and Meininger, 1987; Huangfu and Anderson, 2005; May et al., 2005). Recently it has been demonstrated that key proteins in the Sonic hedgehog (Shh) signal transduction pathway are localized to primary cilia, including the transmembrane receptor Smoothened and Sufu, a negative regulator of Shh signaling (Scholey and Anderson, 2006). Cilia growth and the transport of protein cargoes occurs through intraflagellar transport (IFT), using the same retro- and anterograde motors found in axonal transport (Rosenbaum and Witman, 2002). Functional IFT machinery has been shown to be critical not only for phenotypically normal Shh signaling (Huangfu et al., 2003) but also for proteolytic processing of the transcription factor Gli3 (Haycraft et al., 2005; Huangfu and Anderson, 2005; Liu et al., 2005; May et al., 2005; Tran et al., 2008), in which the full-length activator form of Gli3 is cleaved to form a transcriptional repressor (Wang et al., 2000).

As Gli3 function is crucial for development of the dorsal telencephalon (Theil et al., 1999; Tole et al., 2000), the question arises as to whether Gli3 processing occurs in primary cilia of forebrain neuroepithelia, and whether defects in IFT could affect this processing and thereby influence forebrain development. Although two ciliary mutants have indicated defects in forebrain development (Herron et al., 2002; May et al., 2005), no thorough 
analysis of the role of primary cilia in forebrain and midbrain development has yet been published. To answer these questions, we have used a hypomorphic allele of the Ift 88 gene called cobblestone. Ift 88 is necessary for the formation and maintenance of cilia in a wide range of tissues (Murcia et al., 2000; Banizs et al., 2005; Haycraft et al., 2005; Haycraft et al., 2007) and organisms (Haycraft et al., 2001; Kramer-Zucker et al., 2005). The cobblestone allele has the advantage that embryos live several days longer than the targeted knock-out mutation of Ift 88 (Murcia et al., 2000), allowing the analysis of telencephalic development. Here we show that primary cilia control not only the anatomical organization of dorsomedial telencephalon, but also the organization of both dorsal-ventral and rostral-caudal telencephalic boundaries.

\section{Materials and Methods}

Transmission and scanning electron microscopy

Transmission electron microscopy. Embryonic day 12.5 (E12.5) mouse embryos were collected in cold PBS and fixed for $10 \mathrm{~min}$ by transcardial perfusion (using a glass micropipette) with $2.5 \%$ glutaraldehyde in $0.1 \mathrm{M}$ PIPES buffer, $\mathrm{pH}$ 7.6, containing 2\% polyvinylpyrrolidone (MW 25000, Merck) for $10 \mathrm{~min}$. After immersion fixation of the embryos for an additional hour in the same fixative, $300 \mu \mathrm{m}$-thick coronal sections of the brain were prepared using a vibratome (D.S.K. Microslicer DTK1000, Dosaka EM). For enhancement of membrane staining, samples were incubated in the alkaline diaminobenzidine hydrochloride medium as described previously (Gorgas, 1984) for $60 \mathrm{~min}$, and postfixed with $1.5 \%$ osmium tetroxide containing $1.5 \%$ potassium ferrocyanide for one hour followed by an additional one hour osmification with $1.5 \%$ osmium tetroxide in $0.1 \mathrm{~m}$ sodium cacodylate buffer. Finally, the slices were stained en bloc in 1\% uranyl acetate for $30 \mathrm{~min}$, dehydrated through a graded ethanol series and embedded in Epon 812 (Fluka 45345). Series of semithin sections were stained with a modified Richardson methylene blue-azure II solution and used for selection of corresponding areas in wild-type and mutant brains. Ultrathin sections were stained with lead citrate and analyzed by electron microscopy using a Zeiss EM 906E.

Scanning electron microscopy. Embryonic heads were fixed overnight at $4^{\circ} \mathrm{C}$ in $2.5 \%$ glutaraldehyde/0.1 м PIPES pH 7.4 and subsequently washed 3 times in $0.15 \mathrm{M}$ PIPES, $\mathrm{pH} 7.4$, at $4^{\circ} \mathrm{C}$. The fixed samples were then embedded in 3\% Agarose and cut into $300 \mu \mathrm{m}$ coronal slices in PBS with a D.S.K. Microslicer. The slices were treated for $1 \mathrm{~h}$ at room temperature with $1 \% \mathrm{OsO}_{4}$, washed 3 times with $0.15 \mathrm{M}$ PIPES pH 7.4, and subsequently dehydrated in an ascending ethanol dilution series $(50 \%, 70 \%$, $90 \%, 100 \%)$. The specimens were dried in a CPD 030 critical point dryer (BAL-TEC AG) using $\mathrm{CO}_{2}$ as a transitional medium, followed by sputter coating (BAL-TEC MED 020 sputter coater) of a $20 \mathrm{~nm}$ Gold film. For scanning electron microscopy (EM), a LEO 1530 field emission scanning electron microscope with a Schottky cathode was used (LEO Elektronmikroskopie).

\section{$N$-ethyl-N-nitrosourea mutagenesis and mouse lines}

All animal experiments were in compliance with the regulations of Baden-Württemberg. We used the strain Mapt $t^{\operatorname{tml}(G F P) K l t}$ (tauGFP) (Tucker et al., 2001), backcrossed to wild-type C57BL/6J mice for over 10 generations. tauGFP males were injected intraperitoneally with 150 $\mathrm{mg} / \mathrm{kg} N$-ethyl- $N$-nitrosourea (ENU) and mated to tauGFP females to generate G1 pedigree-founder males. G1 males were mated to their superovulated G2 daughters (supplemental Fig. 1, available at www. jneurosci.org as supplemental material). G3 litters were examined at E11.0-12.5 using epifluorescent microscopy, with E0.5 as noon of the day of the vaginal plug. Identified mutant G2 males were outcrossed to mice of the inbred strain CBA/J for positional cloning. For other experiments, the mouse strains Ift88 $8^{\text {tm1.1Bky }}$ (Haycraft et al., 2007), Ift $88^{\Delta 2-3 \beta g a l}$ (Murcia et al., 2000), and $X t^{J}$ (Hui and Joyner, 1993) were used and genotyped with standard PCR-based protocols.

\section{Positional cloning}

A panel to detect single nucleotide polymorphisms (SNPs) was constructed from primer triplets, spread evenly across the mouse genome, that specifically amplify SNPs between the C57BL/6 and DBA/2 inbred strains. The two SNP-hybridizing primers in each triplet were recognized by fluorescently labeled primers (Amplifluor SNPs HT Genotyping System for FAM-JOE, Millipore) for use in a Stratagene MX3000P real-time PCR device. Segregating $c b s$ embryos were examined using 100 markers covering the 20 murine chromosomes, looking for SNPs in which homozygous $c b s$ mutants were enriched for a homozygous C57BL/6 result. Primer information is available upon request. SNP analysis identified one marker on chromosome 14 that closely cosegregated with the $c b s$ mutants (see Fig. $2 G$ ). Fine mapping was performed with chromosome 14 SSLP markers (see Fig. 2G) (Dietrich et al., 1994), which were resolved on $12 \%$ acrylamide gels. For Ift 88 sequencing, total mRNA was isolated from E12.5 embryonic brain and reverse transcribed with oligo $(\mathrm{dT})_{12-18}$ and SuperScriptII reverse transcriptase (both Invitrogen). PCRs with Ift88-specific primers were as follows: $5 \mathrm{~min} 95^{\circ} \mathrm{C} ; 39$ cycles: $30 \mathrm{~s} 95^{\circ} \mathrm{C}$, $30 \mathrm{~s} 60^{\circ} \mathrm{C}, 30 \mathrm{~s} 72^{\circ} \mathrm{C} ; 5 \mathrm{~min} 72^{\circ} \mathrm{C}$. Agarose gel-purified PCR products, subcloned into the pCRII-TOPO vector, were sequenced (MWG) with appropriate primers.

\section{Whole-mount analysis}

Whole-mount staining using the $2 \mathrm{H} 3$ monoclonal antibody [Developmental Studies Hybridoma Bank (DSHB), Iowa City, IA] was performed as described (Yamashita et al., 1999). Whole-mount skeletal staining using Alcian blue was performed as described (Brachmann et al., 2007).

\section{Immunohistochemical analysis}

Embryos, sections, and stainings were performed as described (Brachmann et al., 2007). Primary antibodies (Ab): rabbit anti- $\beta$-galactosidase (clone 55976, ICN/Cappel) 1:2000, mouse anti-nestin (Rat 401 clone; BD PharMingen) 1:500, RC2 (DSHB) 1:10, mouse anti- $\beta$ tubulin III (TuJ1 clone; Covance) 1:1500, rabbit anti-phospho-histone H3 (Ser10, rabbit 06-570; Upstate) 1:200, and rabbit anti-Pax6 (AB5409, Millipore) 1:3000. Secondary antibodies as described (Brachmann et al., 2007).

\section{Northern blotting}

Northern blotting was performed as described (Tucker et al., 1996), using total RNA prepared from the brain and fore- and hindlimb tissue of E12.5 embryos, and hybridized with a full-length murine Ift 88 or Gli3 cDNA (RZPD) and an $\alpha$ - tubulin cDNA (Lemischka et al., 1981) as loading control.

\section{Quantitative real time $R T-P C R$}

Whole RNA was extracted from embryonic tissue using the RNeasy Mini Kit (74104 Qiagen) according to the manufacturer's instructions. 1-5 $\mu \mathrm{g}$ of RNA was transcribed into cDNA using oligo $(\mathrm{dT})_{12-18}(0,5 \mu \mathrm{g} / \mu \mathrm{l}$, Invitrogen) or random hexamers (50 mm, Applied Biosystems) and SuperScript II RNase $\mathrm{H}^{-}$reverse transcriptase (Invitrogen). Quantitative real time PCRs were performed using the ABI Prism 7000 Detection System (Applied Biosystems) using TaqMan Gene Expression Assays (Applied Biosystems) with $1 \mu \mathrm{l}$ of cDNA (20 $\mu$ l of RTase reactions using 1-5 $\mu \mathrm{g}$ of whole RNA input). The following TaqMan Assays were used: Ift88 (Mm00493675_m1), Wnt7b (Mm00437357_m1), Axin2 (Mm01265783_m1), Ptch1 (Mm00436031_m1), and Gli1 (Mm00494654_m1). The standard quantification protocol was applied with the following cycles: $2 \mathrm{~min}$ at $50^{\circ} \mathrm{C}, 10 \mathrm{~min}$ at $95^{\circ} \mathrm{C}$, followed by 45 cycles: $15 \mathrm{~s}$ at $95^{\circ} \mathrm{C}$ and $1 \mathrm{~min}$ at $60^{\circ} \mathrm{C}$. Each individual reaction was performed in triplicate. GAPDH primers (Mm99999915_g1) were used to normalize results.

Statistical analysis was performed as follows: Relative expression (RE) levels were calculated with the function ( $\mathrm{RE}=2^{-\Delta \Delta \mathrm{Ct}}$ ), where $\Delta \Delta \mathrm{Ct}$ is the normalized difference in threshold cycle $(\mathrm{Ct})$ number between wildtype and $c b s / c b s$ samples, calculated from the mean Ct value of triplicate replicates of any given condition. The mean of RE reported in Figure 7 was calculated from the individual RE values from 4 to 8 independent experiments, and the SEM was calculated from the standard deviation of the 4-8 RE values. Statistical significance was evaluated by applying the Student's $t$ test to the 4-8 RE values, comparing vehicle to TSA treatment. Application of Student's $t$ test to the original $\Delta \Delta \mathrm{Ct}$ values produced comparable $\mathrm{p}$ values. 


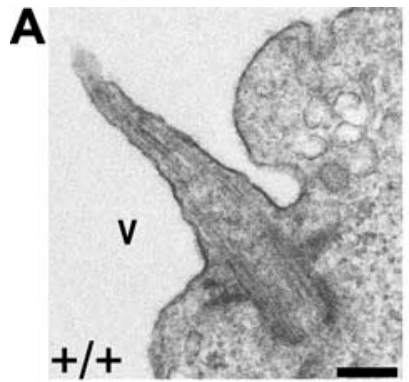

B

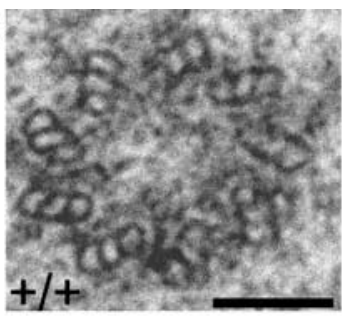

E
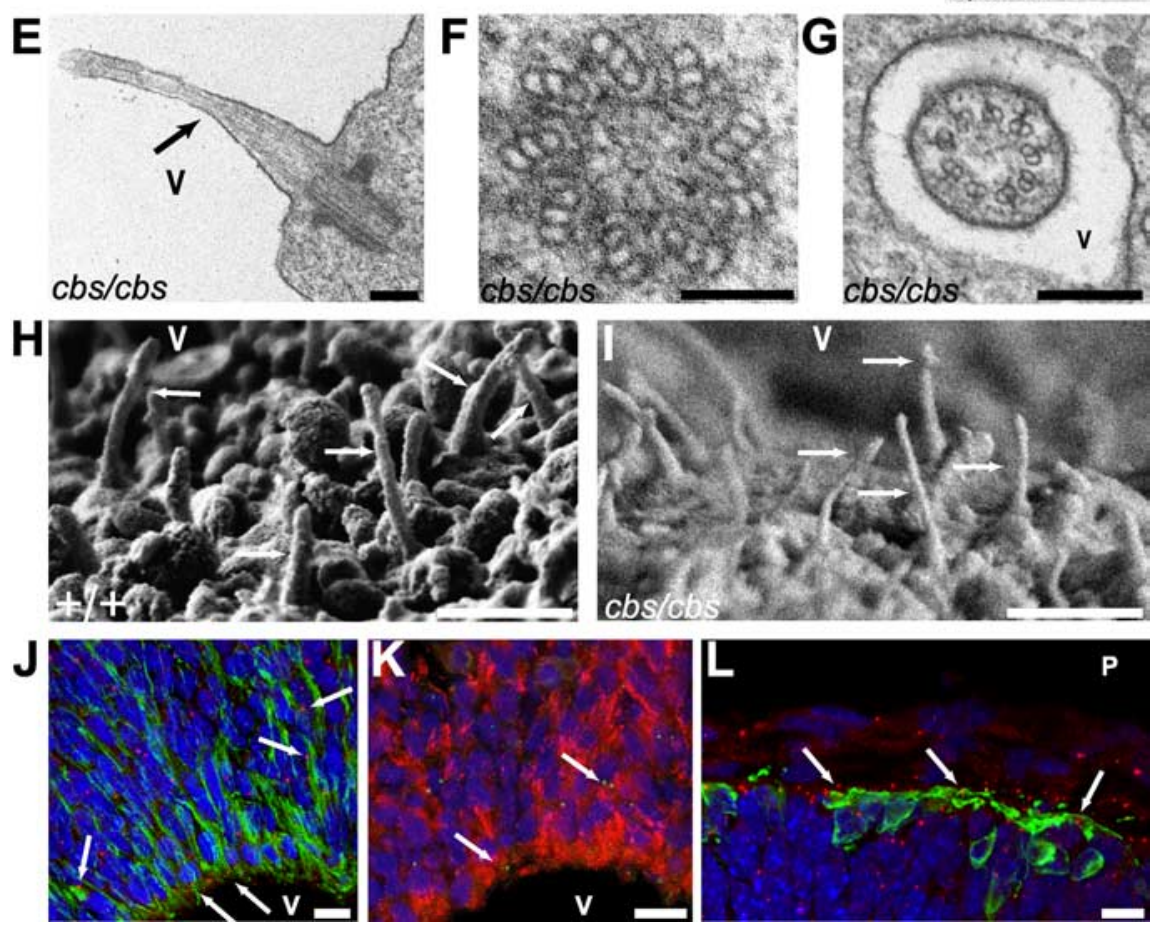

Figure 1. Primary cilia project into the ventricle of embryonic forebrain. $A-I$, TEM $(\boldsymbol{A}-\boldsymbol{G})$ and $(\boldsymbol{H}, \boldsymbol{I})$ scanning $E M$ of cilia projecting into the dorsolateral telencephalic ventricles (V) of 12.5 wild-type $(\boldsymbol{A}-\boldsymbol{D}, \boldsymbol{H})$ and $\operatorname{cbs} / \mathrm{cbs}(\boldsymbol{E}-\boldsymbol{G}, \boldsymbol{I})$ embryos. $\boldsymbol{A}, \boldsymbol{E}$, Cilium cut longitudinally. $\boldsymbol{B}-\boldsymbol{D}, \boldsymbol{F}, \boldsymbol{G}$, Cross sections of ventricular cilia, revealing the basal body $(\boldsymbol{B}, \boldsymbol{F})$, characteristic " $9+0$ " ciliary morphology $(\boldsymbol{C}, \boldsymbol{G})$, and tapering to a " $2+0$ " structure $(\boldsymbol{D})$. $\boldsymbol{E}$, Proximal-to-distal tapering can clearly be seen (arrow). $\boldsymbol{H}, \boldsymbol{I}$, Arrows indicate cilia. $J-L$, Immunofluorescence analysis of Ift88 expression in dorsolateral telencephalon of E11.5 Iff88 $8^{\Delta 2-3 \beta \text { gal }}$ embryos, using an antibody recognizing $\beta$-galactosidase (J, L, red; $\mathbf{K}$, green), which is expressed from the /ft88 locus. The ventricular (V) and pial $(P)$ surface is at bottom $(\boldsymbol{J}, \boldsymbol{K})$, and top $(\boldsymbol{L})$ of the panels, respectively. Arrows indicate characteristic somatic $\beta$-galactosidase deposits in cells colabeled with the following markers: Nestin- (J, green) and RC2-positive (K, red)VZ cells and newborn neurons (L, green, TuJ1 antibody). J-L, Blue, DAPI-labeled nuclei. Scale bars: A, C, E, G, $200 \mathrm{~nm} ; \mathbf{B}, \mathbf{D}, \mathbf{F}, 100 \mathrm{~nm} ; \boldsymbol{H}, \mathbf{I}, 1 \mu \mathrm{m} ; \mathbf{J}, \mathbf{K}, \mathbf{L}, 10 \mu \mathrm{m}$.

\section{Western blotting}

Western blotting was performed as described (Tucker et al., 2001) with a rabbit anti-Gli3 antibody (1:1000, s.c.-20688, Santa Cruz Biotechnology), a goat anti-Ift88 antibody [1:1000 (Pazour et al., 2002)], and a mouse anti- $\beta$-actin antibody (1:5000; clone AC-15, Sigma). HRPconjugated secondary antibodies: mouse anti-goat (1:10,000; cs-2354, Santa Cruz Biotechnology), goat anti-rabbit $(\mathrm{H}+\mathrm{L} ; 1: 10,000, \mathrm{KPL})$, or goat anti-mouse IgG $(\mathrm{H}+\mathrm{L} ; 1: 10,000, \mathrm{KPL})$.

\section{In situ hybridization}

For all in situ hybridizations presented in Figures 4-8, a minimum of 12 $c b s / c b s$ and 14 wild-type littermates were examined from the rostralmost cortex through the beginning of the hindbrain, and the relevant sections are presented. In situ hybridization on paraffin sections was performed as described (Theil, 2005) with following probes: Dbxl (Yun et al., 2001); Foxd1 (Hatini et al., 1996); FoxG1 (Tao and Lai, 1992); Ttr1 (Duan et al., 1989); EphB1 (kind gift from D. Wilkinson, National Institute for Medical Research, London, UK); Lhx2 (Porter et al., 1997); Wnt2b, Pax6, Ngn2, Emx1, Emx2 (Kuschel et al., 2003); Dlx2, Axin2, Reelin, Wnt7b (Theil, 2005); Wnt8b (kind gift from J. Mason, University of Edinburgh,

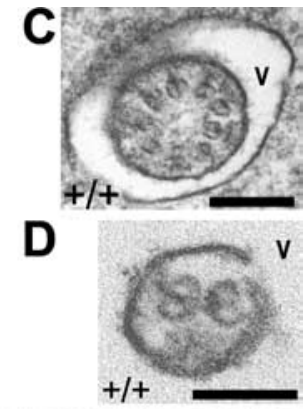

Edinburgh, UK); Shh (kind gift from M. Treier, European Molecular Biology Laboratory, Heidelberg, Germany); Gli3 (kind gift from A. Joyner, Skirball Institute, New York, NY), and Ptch1 (Goodrich et al., 1996).

\section{Luciferase assays}

Primary fibroblast cultures were prepared from E12.5 embryos as described (Tucker et al., 1997). $2 \times 10^{6}$ cells were electroporated (Amaxa nucleofection device, program A23) with a mixture of $5 \mu \mathrm{g}$ of a Shh-responsive firefly luciferase-expressing plasmid (Sasaki et al., 1997) and 300 ng of a Renilla luciferase reporter plasmid (pRL-TK, Promega) to control for transfection efficiency. Cells were plated into 20 wells of a 24-well plate and allowed to grow $36-48 \mathrm{~h}$ to confluency. Medium was shifted to $0.5 \%$ fetal calf serum for $36 \mathrm{~h}$, to allow for the production of cilia, as described (Ocbina and Anderson, 2008). Recombinant murine sonic hedgehog ( $1 \mu \mathrm{g} / \mathrm{ml}, \mathrm{R} \& \mathrm{D}$ Biosystems $)$ was then added for $12 \mathrm{~h}$, and cells were subsequently lysed for luciferase analysis using the DualLuciferase assay system (Promega) with a Veritas Microplate luminometer (Turner BioSystems). All assays were performed at least six times in five-fold replication for each experimental variable; background values were determined with lysates of untransfected cells; and firefly luciferase values were normalized with the Renilla luciferase readouts. Statistical analysis was performed using Student's $t$ test.

\section{Results}

Primary cilia project into the ventricle of developing forebrain

Primary cilia, protruding into the ventricles, have been reported to be present on neuroepithelial cells of the developing cortex (Nagele and Lee, 1979; Cohen and Meininger, 1987; May et al., 2005). These observations were confirmed using transmission electron microscopy (TEM) upon coronal sections of E12.5 brain. Cilia, with lengths varying between $0.5-2 \mu \mathrm{m}$, projected into the ventricle (Fig. $1 A$ ). Transverse sections revealed well defined basal bodies (Fig. $1 B$ ) and a " $9+0$ " morphology in the proximal cilium, confirming them as primary cilia (Fig. $1 C)$. The cilia displayed a proximal-to-distal reduction in the number of microtubule doublets, coming eventually to a " $2+0$ " morphology at the tip (Fig. 1D,E), as reported previously (Cohen and Meininger, 1987). Scanning EM upon E12.5 embryos revealed cilia projecting into the ventricle from dorsal and lateral cortex (Fig. $1 \mathrm{H}$ ), the hippocampal anlage, choroid plexus, and the ganglionic eminences (GEs), with lengths varying between 0.5-3 $\mu \mathrm{m}$. To determine whether neural precursor cells expressed components of the intraflagellar transport (IFT) machin-

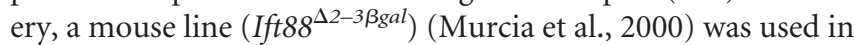
which the lacZ cDNA has been inserted into the locus encoding Ift88, a component of the B complex particles of the IFT machinery (Rosenbaum and Witman, 2002). Examination of E11.5 heterozygous Ift88 ${ }^{\Delta 2-3 \beta g a l}$ embryos revealed expression of Ift88::lacZ by nestin-positive (Fig. $1 J$ ) and RC2-positive (Fig. $1 K$ ) neural precursor cells. Finally, newly born neurons located in the subpial 
mantle zone, identified using the TuJ1 antibody (Moody et al., 1989), were also shown to express Ift88 (Fig. $1 L$ ).

\section{The cobblestone mutant is a}

hypomorphic allele of the Ift 88 gene

The cobblestone ( $c b s)$ mutant was generated in a ENU mutagenesis screen to identify defects in nervous system development. A mouse line (tauGFP) was used in which green fluorescent protein (EGFP) expression is restricted to newborn neurons (Tucker et al., 2001). tauGFP males were injected with ENU and used to establish separate G1 pedigrees (supplemental Fig. 1, available at www.jneurosci.org as supplemental material). Each G1 male was treated as a different potential heterozygote carrier and mated to his $\mathrm{G} 2$ daughters to uncover recessive mutations. The resulting G3 litters were screened with a fluorescent microscope for defects in neurogenesis and nerve development. The $c b s$ mutation was identified by the presence of EGFP-expressing bulges protruding from the pial surface of the E11.0 forebrain (Fig. $2 D)$, lending the brain surface a resemblance to a cobblestone-paved street. The following criteria were used to classify the heterotopias: In cross-section, these heterotopic structures were (1) $50-150 \mu \mathrm{m}$ in diameter, (2) "rosette"-shaped, (3) with a central lumen, (4) had an epithelial-like cell layer lining the lumen, whose cells stained positive for the neural precursor marker nestin (Fig. $3 I, J$ ), and (5) an outer layer with EGFP-expressing cells (Fig. 2D). The EGFP-expressing cells were confirmed as newborn neurons by their expression of $\beta$-tubulin III (Fig. $2 E$ ) and the $165 \mathrm{kDa}$ axonal marker neurofilament (Fig. $2 F)$. The telencephalon itself was shortened along its rostral-caudal axis, while the midbrain was elongated (Fig. $2 A)$. cbs segregated as a recessive mutation (225 mutants/1005 embryos, 140 litters) with no heterozygous phenotype. The mutation was not influenced by the presence of the tauGFP locus, as seen after segregation of the tauGFP marker. $c b s$ mutants showed $8 \%$ and $70 \%$ mortality by E12.5 and E14.5, respectively. $10 \%$ of $c b s$ mutants at E12.5 displayed exencephaly (Fig. $2 A)$. In addition, $c b s$ mutants displayed polydactyly on both fore- (Fig. 2C) and both hind-limbs (Fig. $2 B$ ), and bilateral coloboma (Fig. $2 A$ ). Both of these phenotypes showed $100 \%$ penetrance $(n=225)$. In addition, examination of cardiovascular and pulmonary development between E12.5 and E16.5 uncovered a large number of malformations that may contribute to embryonic lethality, including interven-
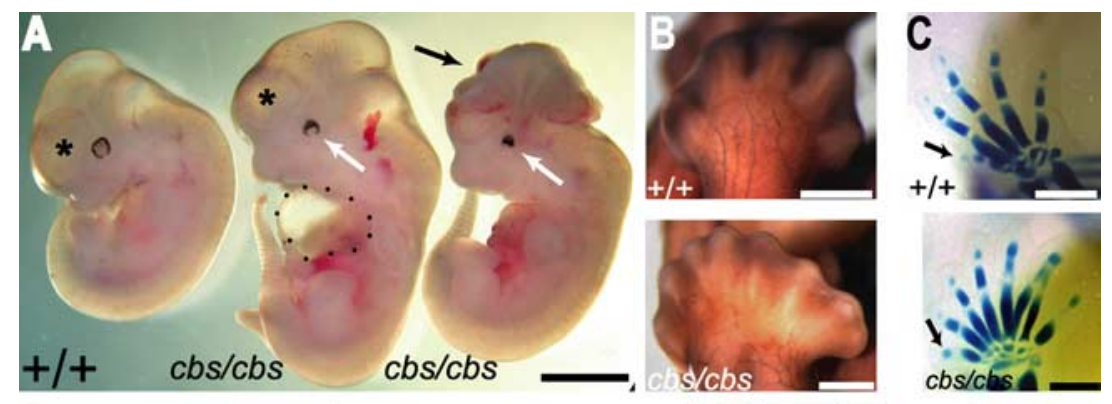
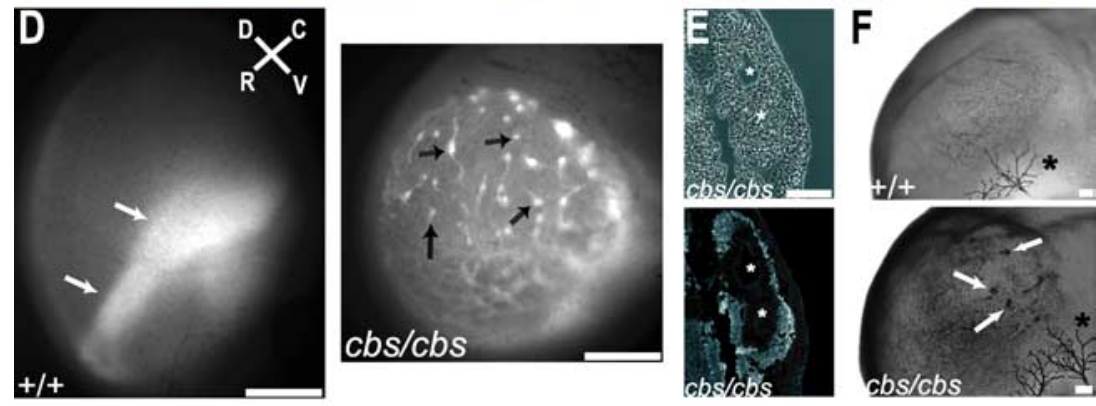

G

$\mathrm{H}$
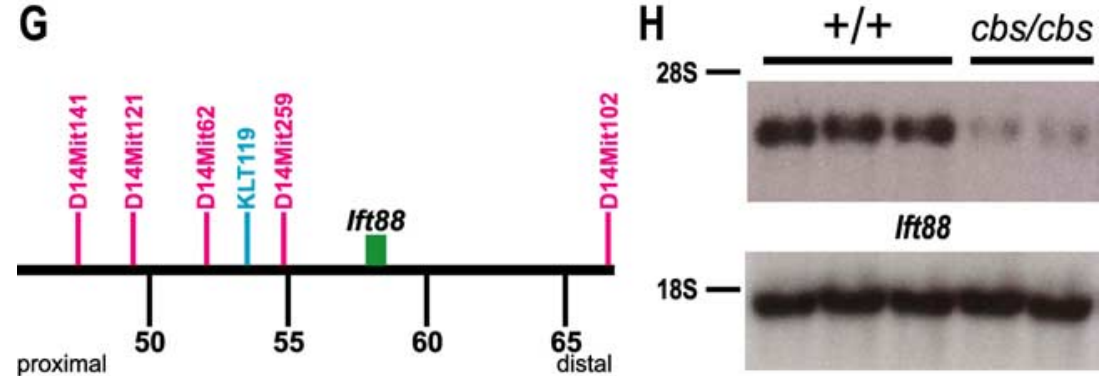

$\alpha-$ tubulin
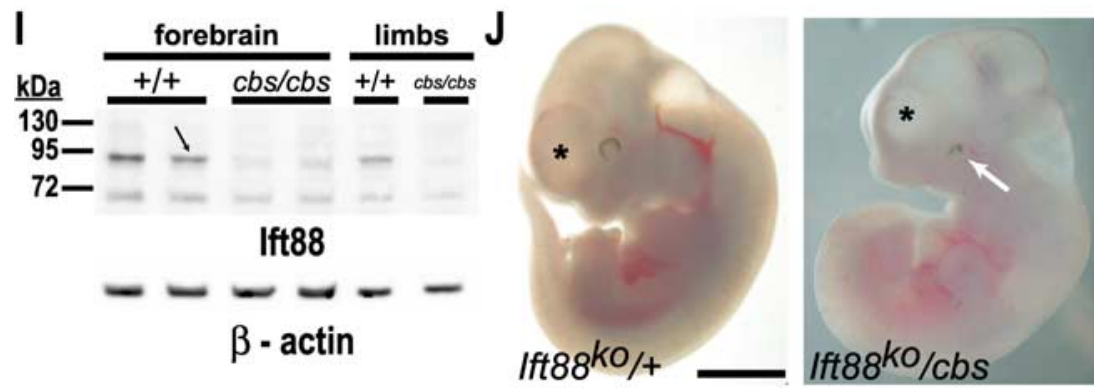

Figure 2. The cobblestone mutant is a hypomorphic allele of the Ift88 gene. $A, E 11.5$ cbs/cbs mutants display a cortex (asterisk) shortened in the rostral-caudal axis (middle embryo), compared with wild-type littermates (left embryo). 10\% of cbs/cbs mutants display exencephaly (right embryo, black arrow). cbs/cbs embryos display colobomas (white arrow), and an enlarged pericardial sac is often observed (black dots). $\boldsymbol{B}, \boldsymbol{C}$, cbs/cbs embryos display bilateral polydactyly on hindlimbs ( $\boldsymbol{B})$ and forelimbs (C). B, E13.5 embryos stained with an anti-165 kDa neurofilament antibody, showing hindlimb innervation. $\boldsymbol{C}$, Alcian blue-stained E14.5 embryos, showing skeletal formation. Arrow indicates pollex. $\boldsymbol{D}$, Whole-mount epifluorescence of E12.5 tauGFP cortex, looking down upon the pial surface. A wild-type embryo shows EGFP-signal where the lateral edge of the cortex folds over, allowing multiple layers of newborn neurons to be seen as a broad stripe of signal (left panel, arrow). In cbs/cbs mutants, heterotopias appear as 40-180 $\mu \mathrm{m}$ wide spheres (right panel, arrows), often with long trails of green signal corresponding to outgrowing axon bundles. Rostral (R), caudal (C), dorsal (D), and ventral (V) axes are indicated. $\boldsymbol{E}$, Cross section of two subpial heterotopias in E11.5 cbs/cbs cortex. Asterisk indicates lumen of each heterotopia. Top, Phase contrast. Bottom, Stained with TuJ1 antibody, indicating newborn neurons in the heterotopia periphery. $\boldsymbol{F}$, Anti-165 kDa neurofilament antibody staining of E11.5 forebrain. Arrows indicate heterotopias in cbs/cbs cortex. The smaller number of stained heterotopias, compared with $(\boldsymbol{D})$, is due to reduced antibody penetration. Asterisk indicates Nervus ophthalmicus. G, Positional cloning of cbs. Distance from the centromere in million bp (black), the SNP marker used for rough mapping (blue), SSLP markers used for fine mapping (red), and the Ift88 gene (green box) are indicated. $\boldsymbol{H}$, Northern blots of whole RNA from whole brain of E12.5 wild-type $(+/+)$ and $c b s / c b s$ embryos. Full-length Ift88 (top) and $\alpha$-tubulin CDNAs (bottom) were used as probes. Ribosomal RNA markers are indicated (left). II, Western blots of protein from forebrain and fore- and hindlimbs (limbs) ofE12.5 wild-type $(+/+$ ) and cbs/cbs embryos. An anti-N-terminal-Ift88 antibody (top), and an anti- $\beta$-actin antibody (bottom) as a loading control, were used. An arrow indicates the Ift88 band. Protein markers (kDa) are indicated (left). J, E11.5 heterozygous Ift88 knock-out embryos $\left(\right.$ Ift $88^{k o} /+$ ) display a wild-type telencephalon (asterisk) with an elongated rostral-caudal profile (cf. $\boldsymbol{A}$, $+/+$ embryo). A compound heterozygote of the Ift88 deletion allele $\left(I f t 88^{k o}\right)$ and the cbs allele $\left(I f t 88^{k o} / c b s\right)$ displays coloboma (white arrow) and a rostro-caudally shortened cortex (asterisk). Scale bars: $\boldsymbol{A}, \boldsymbol{J}, 1.5 \mathrm{~mm} ; \boldsymbol{B}, \boldsymbol{C}, \boldsymbol{D}, 0.5 \mathrm{~mm} ; \boldsymbol{E}, \boldsymbol{F}, 100 \mu \mathrm{m}$. 

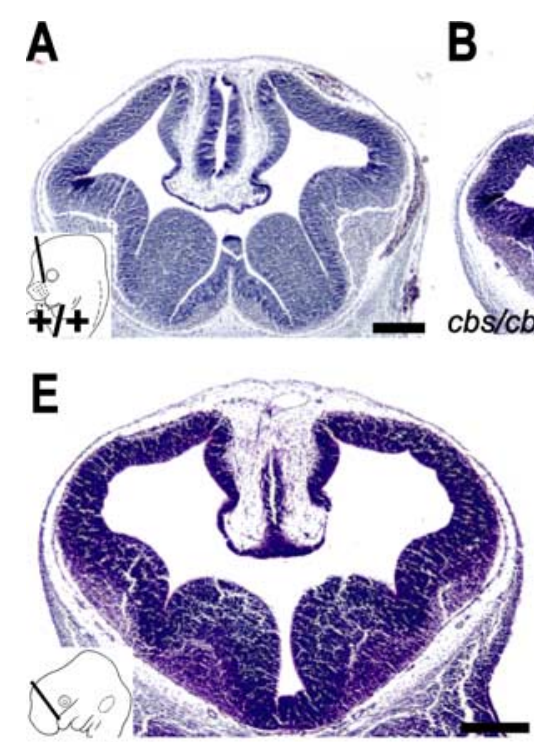

Ift88 ${ }^{\mathrm{ko} /+}$

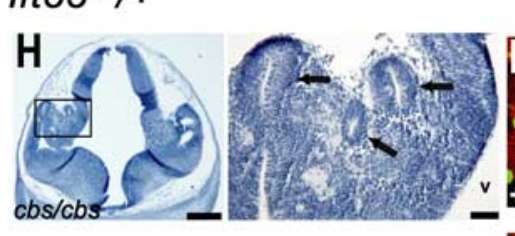

B

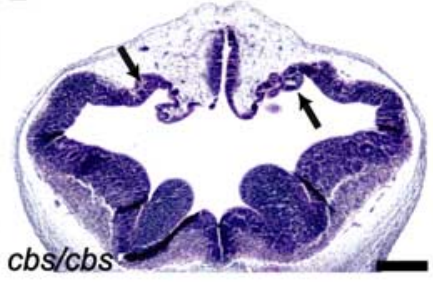

F

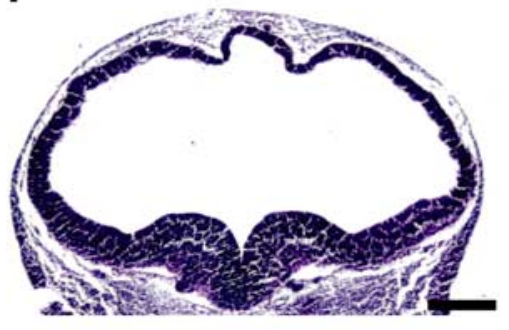

lft8 8 ko/cbs
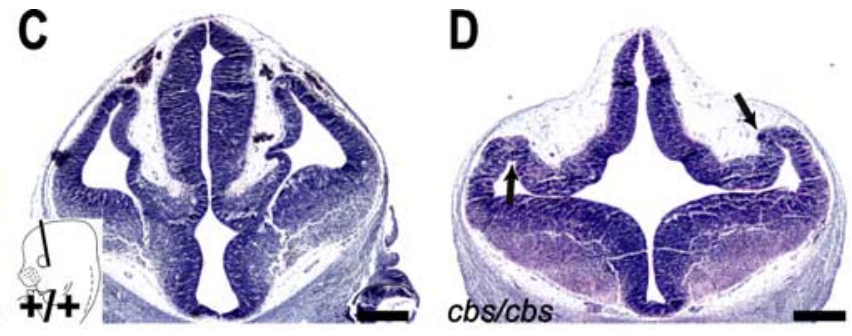

G

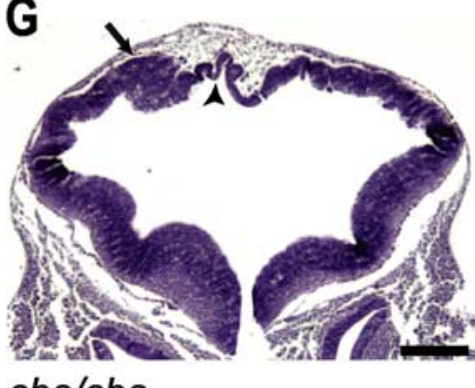

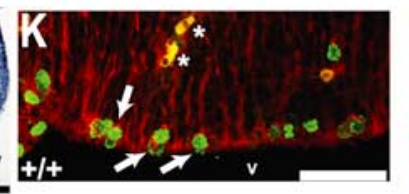
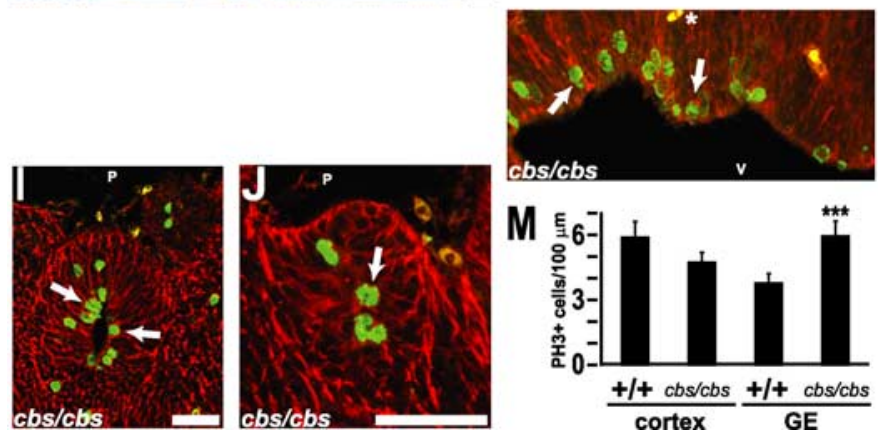
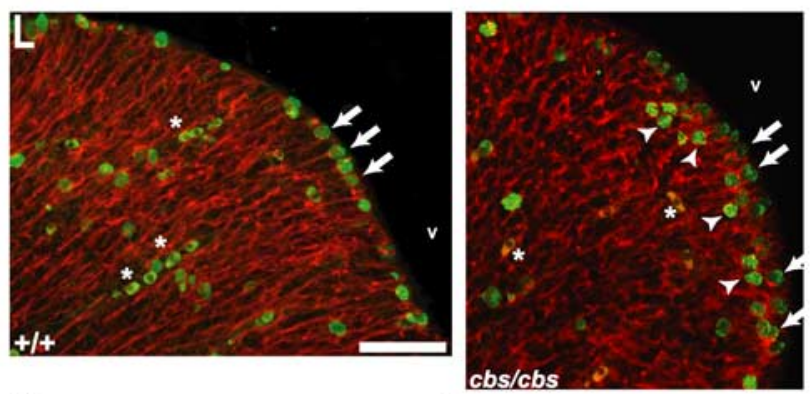

N

0
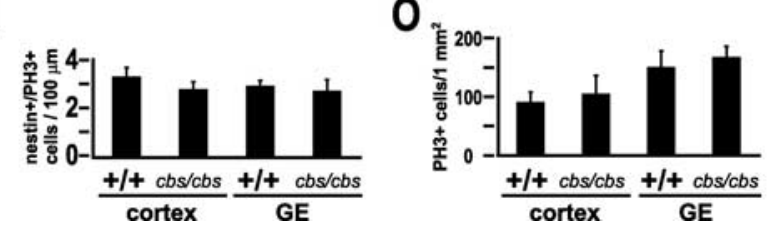

Figure 3. The cbs mutant exhibits a pronounced disorganization of the dorsal telencephalon. $\boldsymbol{A}-\boldsymbol{D}, \boldsymbol{H}$, Hematoxylin-stained coronal sections of E12.5 wild-type $(\boldsymbol{A}, \boldsymbol{C})$ and $c b s / c b s(\boldsymbol{B}, \boldsymbol{D}, \boldsymbol{H}-\boldsymbol{J})$

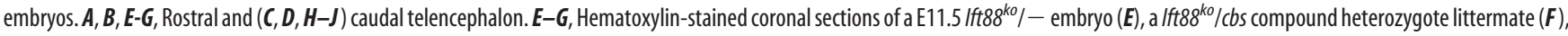
and a $c b s / c b$ s embryo $(\boldsymbol{G}) . \boldsymbol{A}, \boldsymbol{C}, \boldsymbol{E}$, Inset (lower left) indicates the plane of section for $(\boldsymbol{A}, \boldsymbol{B}),(\boldsymbol{C}, \boldsymbol{D})$, and $(\boldsymbol{E}-\boldsymbol{G})$, respectively. $\boldsymbol{H}$, Caudal-most telencephalon showing lateral heterotopias (left, boxed). Enlargement of the boxed region shows them to have a rosette-like morphology (right). $\boldsymbol{B}, \boldsymbol{D}, \boldsymbol{G}, \boldsymbol{H}$, Arrows indicate heterotopias. $\mathbf{G}$, Arrowhead indicates VZ. $\boldsymbol{I}-\boldsymbol{L}$, Mitotic cells revealed with an anti-phosphorylated-histone $\mathrm{H3}$ antibody (green, PH3) in subpial heterotopias $(\boldsymbol{I}, \boldsymbol{J})$ and VZ of the dorsolateral cortex $(\boldsymbol{K})$ and the ganglionic eminences $(\boldsymbol{L})$ in E12.5 wild-type and cbs/cbs embryos. Red = anti-nestin antibody. I-L, Arrows indicate nestin/PH3-double positive cells. Arrowheads indicate sub-VZ mitoses. Asterisks indicate blood cells. $M, N, \mathbf{O}, \mathbf{Q u a n t i t a t i o n ~ o f ~ P H 3 ~ s t a i n i n g ~ i n ~ t h e ~}$ cortex (cortex) and ganglionic eminences (GE), expressed as the number of PH3-positive $(\boldsymbol{M})$ and double nestin/PH3-positive $(\boldsymbol{N})$ cells per $100 \mu \mathrm{m}$ of VZ, and in the number of basally located (i.e., $>30 \mu \mathrm{m}$ from the ventricular zone) PH3-positive cells per $1 \mathrm{~mm}^{2}(\mathbf{O}) .{ }^{* * *} p<0.001$, Student's $t$ test. $A-L$, Dorsal is to the top. $\boldsymbol{H}-\boldsymbol{L}$, Lateral is to the left; $V$, ventricle; $P$, pial surface. Scale bars: $A-G$, $\boldsymbol{H}$ (left), $300 \mu \mathrm{m} ; \boldsymbol{H}$ (right), $\boldsymbol{I}-\boldsymbol{L}, 50 \mu \mathrm{m}$.

tricular and interatrial septal defects, persistent truncus arteriosus, arteria lusoria, and pulmonary aplasia/hypoplasia (described in detail in supplemental Fig. 2, available at www.jneurosci.org as supplemental material).

To perform positional cloning, the $c b s$ mutant was crossed to wild-type mice of the CBA/J background, F1 progeny were intercrossed, and genomic DNA from the resulting F2 embryos was analyzed with strain-specific SNP primers. This approach identified one marker on chromosome 14 cosegregating with $c b s$ mutants (Fig. 2G). Fine mapping was performed using standard SSLP markers (Dietrich et al., 1994), and thereby positioned the cbs locus $0.5 \mathrm{cM}$ distal to the marker D14Mit259 (Fig. 2G). Examination of candidate genes revealed one, Ift 88 , that had been previously reported to show both polydactyly (Zhang et al., 2003) and defects in neural tube formation (Murcia et al., 2000) when mutated. Northern blot analysis performed upon RNA isolated from 12.5 d.p.c brain identified a single transcript in both wildtype and cbs/cbs embryos (Fig. $2 H$ ). Quantitation revealed a $66.7 \pm 2.0 \%$ decrease in the levels of Ift $88 \mathrm{mRNA}$ in $c b s / c b s$ brain. Western blot analysis of protein levels of Ift 88 in whole brain, using an anti-N-terminal-Ift 88 antibody, revealed a single band of $\sim 90 \mathrm{kDa}$ in both wild-type and $c b s / c b s$ embryos (Fig. $2 I$ ). Quantitation revealed a 75.0 $\pm 3.0 \%$ decrease in the levels of Ift 88 protein in $c b s / c b s$ brain $(n=5, p<0.01$, Student's $t$ test $)$. Analysis of mRNA and protein levels in fore- and hind- limbbuds with Northern blots, quantitative real-time RT-PCR, and Western blots using anti-Ift 88 antibodies directed against either the $\mathrm{N}$ terminus (Fig. $2 I$ ) or the $\mathrm{C}$ terminus demonstrated a similar reduction in protein levels (supplemental Fig. 3C, available at www. jneurosci.org as supplemental material). 
To determine whether the genetic defect in the $c b s$ mutant is located in the Ift 88 gene, we performed a complementation analysis by crossing $c b s$ heterozygotes to mice heterozygous for a targeted deletion of the Ift 88 gene (Ift8 $8^{\text {tm1.1Bky }}$ ) (Haycraft et al., 2007). 7 compound heterozygotes for both alleles were identified by PCR-based genotyping, and they displayed the morphological characteristics of $c b s$ homozygotes, including bilateral coloboma, a rostro-caudally shortened telencephalon, and an enlarged midbrain (Fig. $2 J$ ). Interestingly, compound heterozygotes displayed a rightward-looping heart tube, an indication of situs inversus, in some cases (3/7 cbs/Ift88 ${ }^{\text {tm1.1Bky }}$ embryos; supplemental Fig. 4, available at www.jneurosci.org as supplemental material). In contrast, $c b s / c b s$ mutants never displayed situs inversus $(n=61)$. Of 48 littermate embryos genotyped as wild type or heterozygous for either the Ift 88 or the $c b s$ mutation, none showed the phenotypes reported above. We conclude from these data that the two mutations do not complement one another and that $c b s$ is a hypomorphic allele of Ift88. As sequencing of the mRNA transcript in the $c b s$ mutant revealed no changes in the ORF or the $5^{\prime}$ and $3^{\prime}$ UTRs, this further indicates a mutation in an intron or a regulatory region.

\section{Cilia are present in the ventricles of $c b s$ mutants}

Previous reports of Ift 88 mutants (Murcia et al., 2000; Haycraft et al., 2001, 2005, 2007; Kramer-Zucker et al., 2005; Banizs et al., 2005) have shown that cilia are either not formed or not maintained. Surprisingly, TEM on coronal forebrain sections from E12.5 $\mathrm{cbs} / \mathrm{cbs}$ embryos revealed primary cilia projecting into the ventricle that did not appear to be different from those of the wild type (Fig. $1 E-G$ ). The cilia derived from basal bodies (Fig. $1 F$ ), demonstrated a " $9+0$ " morphology (Fig. 1G), and tapered in a proximal-to-distal manner (Fig. 1E). Using scanning EM, cilia could also be detected projecting into the ventricle from the dorsolateral telencephalon (Fig. $1 I$ ) and the GEs of $c b s / c b s$ mutants. The cilia in the dorsolateral telencephalon of the $c b s$ mutant appeared of normal length (wild type: $973 \pm 160 \mathrm{~nm}(n=26)$; cbs/cbs: $894 \pm 213 \mathrm{~nm}(n=33), p=0.25$, Student's $t$ test). Thus, both TEM and scanning EM revealed ultrastructurally normal cilia projecting into the ventricle of $c b s / c b s$ forebrain. In addition, ultrastructurally and morphologically normal cilia were also found in the midbrain (supplemental Fig. 5A, $B$, available at www.jneurosci.org as supplemental material) and bronchia (supplemental Fig. 5C,D, available at www.jneurosci.org as supplemental material) of E12.5 cbs/cbs embryos.

\section{The cbs mutant exhibits a pronounced disorganization of the dorsal telencephalon}

$c b s / c b s$ mutant forebrain demonstrated a major disruption in dorsal telencephalic morphology (Fig. 3A-G). In E12.5 wild-type littermates, the telencephalic midline invaginated and demonstrated development of the choroid plexus, cortical hem, and the hippocampal anlage (Fig. 3A). Although invagination of the telencephalic midline did occur in $c b s / c b s$ mutants, the morphology of the dorsomedial telencephalon was severely affected and the hippocampal primordium and the cortical hem could not be identified morphologically. Instead, $c b s / c b s$ mutants demonstrated a kinked ventricular zone (VZ) that folded in upon itself (Fig. $3 B$, arrows) to form rosette-like heterotopias (Fig. $3 H-J$ ). In contrast, development of the medial and lateral GEs appeared relatively normal (Fig. $3 B$ ). At caudal levels, the diencephalon and the dorsal telencephalon are clearly separated in wild-type embryos (Fig. 3C). This distinction is lost in $c b s / c b s$ mutants, where a continuous $\mathrm{VZ}$ runs from the dorsal diencephalon to the dorsolateral telencephalon (Fig. 3D). An even more severe phenotype was observed in E11.5 embryos bearing both the $c b s$ and the Ift 88 knock-out allele (Fig. $2 \mathrm{~J}$ ). In these embryos, the dorsal telencephalic midline barely invaginated, the cortical VZ was reduced to a very thin strip, and the medial and lateral GEs were also greatly reduced in size (Fig. $3 F$ ). Later developmental stages of this complementation cross could not be examined because of an earlier lethality than that seen in $c b s / c b s$ mutants. Similar to E12.5, E11.5 cbs/cbs mutants demonstrated a reduced invagination and disorganization of the dorsal midline, invaginations of the cortical VZ (Fig. 3G, arrowhead), and subpial heterotopias (Fig. 3G, arrow). However, both the cortex and the GEs were thicker than in the cbs/Ift $88^{\text {tm 1.1Bky }}$ complementation mutant (Fig. 3F).

Intriguingly, in E12.5 cbs/cbs mutants, the number of mitotic cells at the VZ was not significantly altered in the cortex ( $p=$ $0.06, n=5$, Student's $t$ test) (Fig. 3M). Many of the mitotic VZ cells were positive for the anti-nestin antibody, indicating them to be neural precursors (Fig. $3 K, L$, arrows; $N$ ). In contrast, the GEs showed a large increase in mitotic cells (Fig. 3M) because of the appearance of cells dividing 10-30 $\mu \mathrm{m}$ away from the VZ, many of which were nestin-negative (Fig. $3 L$, arrowheads; $N$ ). Similar results were seen at E11.5. In addition, mitotic cells at the lumen of heterotopias could also be identified as nestin-positive (Fig. $3 I, J$ ). Finally, no change was seen for the number of mitotic cells located basally $>30 \mu \mathrm{m}$ from the ventricular zones of the cortex and GE (Fig. 3O).

\section{Dorsomedial telencephalic cell types are specified but do not form morphological structures in $c b s$ mutants}

The dorsomedial telencephalon gives rise to several different cell types including the choroid plexus, the cortical hem, the hippocampus and Cajal-Retzius cells. As histological examination demonstrated a gross disorganization of these structures in $c b s /$ $c b s$ mutants, the determination of these structures was examined with appropriate tissue-specific markers. The choroid plexus forms from the dorsal midline and expresses Ttr1 (Fig. 4A, arrow) (Duan et al., 1989). In $c b s / c b s$ mutants, Ttr1 was expressed but in a patchy pattern and at a considerably lower expression level than wild-type littermates (Fig. 4A). The cortical hem lies directly dorsal to the choroid plexus and is marked by the expression of several Wnt family genes including Wnt $2 b$ (Grove et al., 1998). Examination of $W n t 2 b$ expression in $c b s / c b s$ mutants revealed a domain of expression in the dorsal telencephalon (Fig. $4 B$, arrow) lateral to the Ttr1 expression domain seen in an adjacent section (cf. Fig. 4A). Similar to the Ttr1 expression pattern, $W n t 2 b$ was expressed at lower levels and in scattered groups of cells (Fig. $4 B$ ). The hippocampal anlage lies adjacent to the cortical hem and expresses EphB1 (Tole et al., 2000). In $c b s / c b s$ mutants, however, EphB1 expression was not detected in the dorsal telencephalon, while its expression in the ventral telencephalon was unaffected (Fig. 4C). The hippocampus is further characterized by high level expression of the L $h \times 2$ homeodomain gene (Fig. $4 D)$, which is required for proper hippocampal development (Porter et al., 1997; Bulchand et al., 2001; Monuki et al., 2001; Mangale et al., 2008). In cbs/cbs mutants, however, the high level expression of $L h \times 2$ was diminished (Fig. $4 D$ ), suggesting that the hippocampus was not specified correctly. Finally, the cortical hem is a major source of Cajal-Retzius (CR) neurons, the earliestborn cortical neurons, which are characterized by the expression of reelin (Meyer et al., 2002; Takiguchi-Hayashi et al., 2004). In situ hybridization analysis showed a single layer of reelinexpressing cells at the cortical marginal zone of E12.5 wild-type 


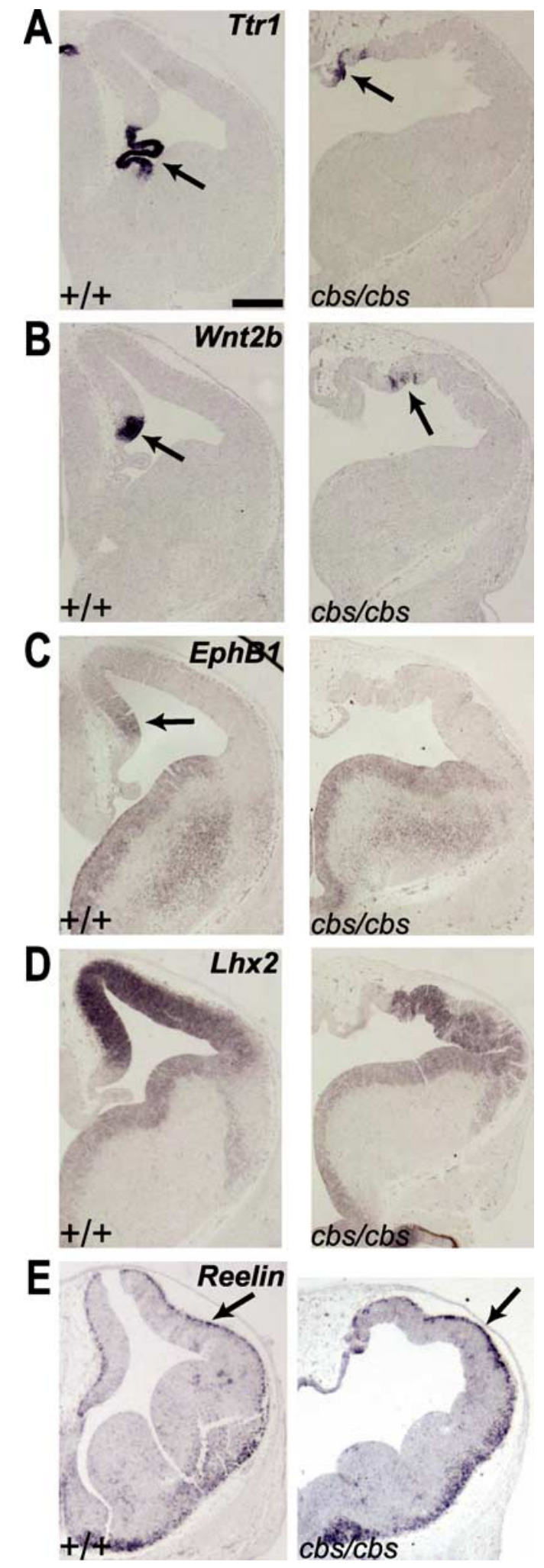

Figure 4. Dorsomedial telencephalic cell types are specified but do not form morphological structures. $\boldsymbol{A}-\boldsymbol{E}$, In situ hybridization analysis of 12.5 wild-type and cbs/cbs embryos. For each coronal section, one telencephalic half is shown, with dorsal to the top, lateral to the right. Arrows indicate signal described in text.A, Ttr1.B, Wnt2b. C, EphB1.D, Lhx2.E, Reelin. Scale bars: $300 \mu \mathrm{m}$.

and $c b s / c b s$ mutant embryos (Fig. $4 E$, arrow). Together, this data indicate that cells characteristic for the dorsomedial telencephalon are formed in the $c b s$ mutant but they fail to form morphologically distinct structures.

\section{The pallial-subpallial boundary does not form properly in} cbs mutants

We next analyzed whether dorsal-ventral subdivisions of the telencephalon formed correctly in $c b s / c b s$ mutants. Pax6 (Fig. 5A) and $N g n 2$ (Fig. $5 B$ ) show a lateral-high to medial-low expression gradient in the developing neocortex, with a sharp expression boundary at the pallial-subpallial boundary (PSPB) of the telencephalon (Walther and Gruss, 1991; Gradwohl et al., 1996). In $c b s / c b s$ mutant telencephalon the graded expression of both genes was lost, and their ventral expression domains were not as sharply delineated as in wild-type embryos (Fig. $5 A, B$ ). Immunofluorescence analysis for Pax6 also revealed scattered Pax6-positive cells at the boundary region. (Fig. 5C). In situ hybridization on adjacent sections for Dlx2, which marks the medial and lateral GEs (Bulfone et al., 1993), further suggested that scattered Pax6 and $\mathrm{Ngn} 2$ expressing cells were located within ventral telencephalic territory (Fig. 5D). Conversely, Dlx2 also showed a diffuse border of expression, and scattered $D l x 2$-expressing cells were found in neocortical areas (Fig. 5D). Consistent with histological analysis, however, Dlx2 expression in the GEs did not differ substantially from that of wild-type littermates (Fig. 5D). To further define the formation of the PSPB in $c b s / c b s$ mutants, we examined the expression of the $D b \times 1$ homeobox gene in the ventral pallium, located just dorsally to the PSPB (Medina et al., 2004). In $c b s / c b s$ mutants, $D b \times 1$-expressing cells were more dispersed, especially within the neocortex (Fig. 5E). Together, these data indicate that the PSPB does not form properly and that cells expressing dorsal or ventral markers intermingle at the boundary.

\section{The dorsal telencephalic-diencephalic boundary in cbs mutants}

As the PSPB does not form correctly in $c b s / c b s$ mutants, it was of interest to see whether the dorsal telencephalic-diencephalic boundary was also affected. Histological analysis had identified a highly abnormal structure in this region containing many of the rosettes (Fig. $3 \mathrm{D}, \mathrm{H}-\mathrm{J}$ ), which we have named the caudal rosetterich area (CRA). Because of the severity of these abnormalities, morphological landmarks could not be used, and so we used developmental marker analysis to define the cellular composition of this structure. Foxg1 is expressed by all telencephalic cells, except for the cortical hem and CR neurons, and shows an expression gradient in the hippocampus with lower expression levels medially (Fig. 6A) (Tao and Lai, 1992; Hanashima et al., 2002). In situ hybridization for Foxg1 revealed a similar pattern in cbs/cbs mutant neocortex, with higher expression levels laterally and weak expression medially, but showed an absence of Foxg1 expression in the CRA except for a small patch of cells located at its lateral margin (Fig. 6A). Because of lower Foxg1 expression levels in developing hippocampus of wild-type embryos we analyzed the expression patterns of $L h \times 2$ and $E m \times 2$, which both show an opposite expression gradient to Foxg1 in the cortex (Fig. $6 B, C)$. In addition to their expression in the $c b s / c b s$ neocortex (Lhx2: Fig. 4D; Emx2: Fig. 8C) both $L h \times 2$ and Emx2 showed a scattered expression within the CRA (Fig. $6 B, C$ ). Similarly, inspection of $W n t 2 b$ and Ttr 1 expression, which mark the cortical hem and choroid plexus in wild-type embryos, respectively, revealed the presence of scattered Wnt2b- (Fig. 6D) and Ttr1- (Fig. $6 E$ ) positive cells in the lateral-most part of the CRA. These data suggest that telencephalic cells contribute to the CRA.

However, Emx2 and $L h \times 2$ are also expressed in the eminentia thalami (ET) and in the dorsal diencephalon, respectively. We therefore investigated the possibility that the CRA also contains diencephalic cell types. Shh expression at the zona limitans in- 
trathalamica (ZLI) separates the dorsal from the ventral thalamus in both wildtype and $c b s / c b s$ mutant forebrains (Fig. $6 F$ ), indicating that the $c b s$ mutant dorsal thalamus does not extend into the CRA. Consistent with this, Dlx2 expression, which marks the ventral thalamus (VT) of wild-type embryos (Bulfone et al., 1993), is found in the CRA of $c b s$ mutants, although in a highly disorganized and patchy manner (Fig. 6G). Finally, Foxd 1 is expressed in the ventral diencephalon of wild-type embryos (Fig. $6 H$ ). Similar to $D l \times 2$, a group of Foxd1-expressing cells is located within the CRA of $c b s / c b s$ mutants (Fig. $6 H$ ). However, neither Dlx2 (Fig. 6G) nor Foxd1 (Fig. $6 H$ ) expressions were observed in the dorsal telencephalon. Together, these data suggest that the CRA is mainly composed of VT and ET cells, but also contains some scattered telencephalic cells.

\section{Wnt expression and signaling is upregulated in $c b s$ mutants}

Mutations in ciliary and basal body proteins have recently been shown to result in an upregulation of canonical Wnt signaling (Gerdes et al., 2007; Corbit et al., 2008). To examine whether Wnt signaling may play a role in the morphological defects seen in the $c b s$ mutant, we examined the expression of several Wnt genes. In the wild-type E12.5 neocortex, Wnt7b expression can be detected in the cortical hem and hippocampal VZ, and in cortical neurons, but it is absent from the neocortical VZ (Fig. 7A). While $c b s / c b s$ mutants displayed normal $W n t 7 b$ expression in cortical neurons, they showed ectopic expression in isolated neocortical progenitor cells, similar to Gli3 mutants (Fig. 7A) (Theil, 2005). Interestingly, at caudal levels of $c b s / c b s$ forebrain, strong $W n t 7 b$ expression could be detected in the CRA, which likely corresponds to its expression in the wild-type VT and ET (Fig. 7B). A similar but not as extensive upregulation was also observed for $W n t 8 b$ in the CRA (Fig. 7D). This upregulated Wnt gene expression in the CRA prompted us to test for activation of canonical Wnt signaling. Axin2, a direct target of the canonical Wnt signaling pathway (Jho et al., 2002; Lustig et al., 2002), has a graded expression in wildtype dorsomedial telencephalon (Fig. $7 E$ ), while $c b s / c b s$ mutants showed a patchy Axin2 activation dorsomedially (Fig. $7 E$ ). In contrast, Axin2 was strongly expressed in the CRA of $c b s / c b s$ mutants, especially within heterotopias (Fig. $7 F$ ). At this level, Axin2 expression also was found in the dorsal thalamus of both genotypes (Fig. 7F). To quantitate these increases in Wnt signaling, we performed quantitative, real-time RT-PCR upon mRNA extracted from E12.5 cbs/cbs telencephalon, comparing gene expression levels to wild-type littermates. We observed a $1.54 \pm$ 0.25 -fold increase in the expression of Axin2 $(p<0.05, n=5$, Student's $t$ test) but curiously not that of Wnt7b (1.25 \pm 0.24 -fold increase, $p=0.37, n=6$ ), indicating that the heterotopic expression of Wnt7b is in aggregate not reflective of an increase in total mRNA levels. Together, these data suggest that an ectopic activa- tion of canonical Wnt signaling in the CRA occurs, but it cannot be directly attributed to an upregulation of $W n t 7 b$ expression.

\section{Targets of Shh signaling and Gli3 protein processing are} disturbed in the forebrain of $c b s$ mutants

Several phenotypes seen in $c b s / c b s$ mutants resemble that seen in the Gli3 deletion mutant $X t^{J}$, including polydactyly, defects in the determination of dorsal telencephalic tissue, the formation of rosette-shaped heterotopias in the dorsal cortex, and the relaxation of the telencephalic-diencephalic boundary (Johnson, 1967; Theil et al., 1999; Tole et al., 2000; Fotaki et al., 2006). This prompted us to examine Gli3 expression patterns in the $c b s \mathrm{mu}-$ tant. High levels of Gli3 expression were retained in the telencephalon of $c b s / c b s$ mutants (Fig. $8 \mathrm{~A}$ ). One of the hallmarks of the $X t^{J}$ mutant is a complete abolishment and a strong downregulation, respectively, in the expression of the transcription factors $E m \times 1$ and Emx2 (Theil et al., 1999; Tole et al., 2000). Intriguingly, examination of both of these markers revealed no downregulation in their expression (Fig. $8 B, C$ ), rather, a patchy Emx1 expression pattern.

Several laboratories have reported defects in the proteolytic processing of Gli3 in a number of IFT mutants (Haycraft et al., 2005; Huangfu and Anderson, 2005; Liu et al., 2005; May et al., 2005; Tran et al., 2008). To examine the effect upon Gli3 processing in the $c b s$ mutant, we performed Western blotting upon protein extracted from 12.5 d.p.c forebrain tissue. Using an anti-Nterminal-Gli3 antibody, the production of both the full-length, 


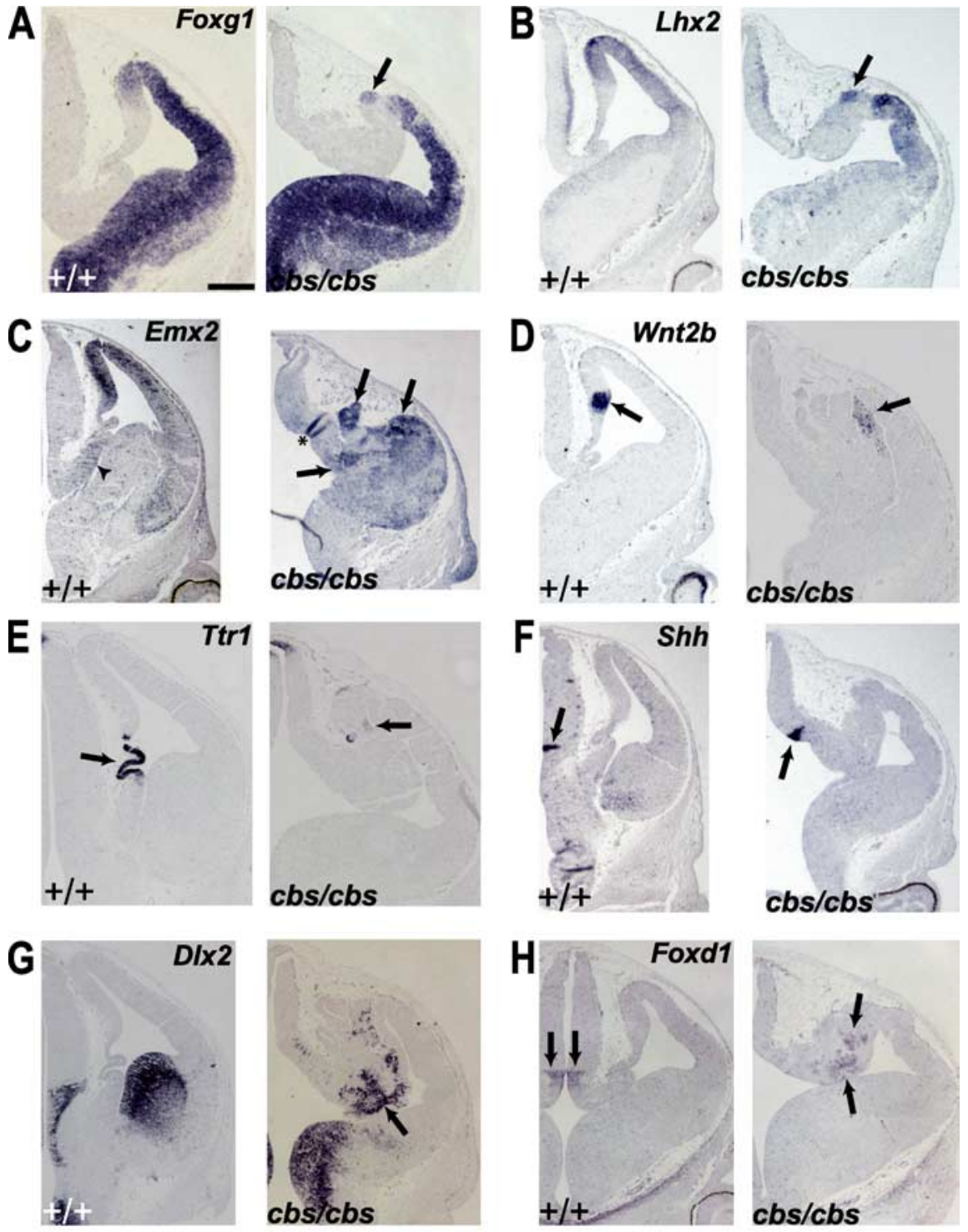

Figure 6. The dorsal telencephalic-diencephalic boundary in the cbs mutant. In situ hybridization analysis of 12.5 wild-type and $c b s / c b s$ embryos. For each coronal section, only one telencephalic half is shown, with dorsal to the top, lateral to the right. $\boldsymbol{A}$, FoxG1. B, Lhx2. C, Emx2. D, Wnt2b. E, Ttr1. F, Shh. G, Dlx2. H, Foxd1. A-H, Arrows indicate signal described in text. C, Asterisk indicates fold in tissue. Scale bars: $300 \mu \mathrm{m}$.

$190 \mathrm{kDa}$ repressor (Fig. $8 \mathrm{~F}$, arrows) form of Gli3 and the truncated, $90 \mathrm{kDa}$ activator form of Gli3 could be detected (Fig. $8 \mathrm{~F}$, arrowheads). In $c b s / c b s$ mutants, no change was observed in the amount of the cleaved isoform (Fig. $8 F$, arrowheads), whereas a strong increase was observed in the amount of the full-length isoform (Fig. 8F, arrows). Quantitation revealed a 5.6-fold increase in the amount of the full-length Gli3-isoform in $c b s / c b s$ mutant forebrain, compared with wild type, whereas the amount of processed-Gli3 isoform in the $c b s / c b s$ mutant did not change significantly (Fig. $8 \mathrm{G}$ ). Together, the total amount of Gli3 protein increases by $67.2 \%$ in $c b s / c b s$ forebrain (Fig. $8 G$ ). These changes are not explained at the level of transcription, as Northern blot analysis of RNA isolated from the forebrain revealed neither a change in the quantity of Gli3 mRNA nor in transcript size (Fig. $8 E)$.

To examine the potential effect of an overproduction of the full-length Gli3 isoform in $c b s / c b s$ forebrain, in situ analysis of Ptch1, a downstream target of Shh signaling (Goodrich et al.,
1996; Marigo et al., 1996; Platt et al., 1997; Agren et al., 2004), was performed. The expression of Ptch1 seemed to increase in the GEs of $c b s / c b s$ mutants (Fig. $8 D$ ), but no expression was observed in the dorsal telencephalon. To quantitate these apparent increases in Ptch1 expression, we performed quantitative, real-time RT-PCR upon mRNA extracted from E12.5 cbs/cbs telencephalon, comparing gene expression levels to wild-type littermates. Indeed, we observed a clear increase in the expression of both Ptch1 and Gli1 (Fig. 8 H), another downstream target of Shh signaling (Lee et al., 1997). To test whether cells in the cbs/ cbs mutant lost their competence to respond to Shh signaling, fibroblast cultures were prepared from decapitated, eviscerated E12.5 wild-type and $c b s / c b s$ embryos. Fibroblasts were electroporated with a Shh-responsive plasmid that expresses the firefly luciferase gene under the control of a minimal promoter and 8 tandem copies of a Gli binding site (Sasaki et al., 1997), using a plasmid expressing Renilla luciferase to control for transfection efficiency. Cells were allowed to reach confluency and the production of cilia was promoted by switching to a low-serum medium (Ocbina and Anderson, 2008), followed by treatment for $12 \mathrm{~h}$ with Shh at $1 \mu \mathrm{g} / \mathrm{ml}$ and lysis for quantitation of luciferase activity. In wild-type fibroblasts, Shh was able to induce a sevenfold increase in luciferase expression from the Gli-responsive reporter plasmid (Fig. 8I), but both basal and Shh-induced luciferase expression levels were greatly reduced in the $c b s / c b s$ fibroblasts (Fig. 8I).

\section{Discussion}

We have uncovered a hypomorphic allele of the intraflagellar transport (IFT) gene Ift 88 and used it to elucidate a critical role for primary cilia in the development of the dorsal telencephalon. cobblestone mutants show severe regionalization defects in the forebrain with a disorganization of the dorsomedial telencephalon including the choroid plexus, the cortical hem and the hippocampus. The mutation also affects the pallial-subpallial boundary and the dorsal telencephalic-diencephalic boundary, major boundaries demarcating the dorsal telencephalon. This forebrain phenotype shows strong resemblance to that of the Gli3 mouse mutant $X t^{J}$ and indeed, proteolytic processing of the Gli3 protein is altered, shifting the relative ratio of Gli3 activator and repressor forms. In addition, the total amount of Gli3 protein is increased by $67 \%$ in the $c b s / c b s$ mutants, explained by the large increase in the fulllength, unprocessed isoform (Fig. $8 F$ ). Since the levels of Gli3 mRNA are not increased in the $c b s / c b s$ mutants, we speculate that this full-length form is stabler within the cell than the processed isoform. All three Gli family members share two conserved sequences in their $\mathrm{C}$ terminals that target them for rapid degradation (Huntzicker et al., 2006). These sequences would also be 
present in the full-length isoform, and thus the reasons for its potential enhanced stability are unclear, but it is possible that motifs in the $\mathrm{N}$ terminus may protect the protein from degradation.

Several lines of evidence indicate that Ift 88 is the defective gene in the $c b s$ mutant. First, fine mapping indicated it to lie in a $0.5 \mathrm{cM}$ interval containing Ift 88 . Second, the Ift $88 \mathrm{mRNA}$ and protein are expressed at only $25 \%$ of the levels of wild-type embryos. Third, compound cbs/Ift $88^{\text {tm } 1.1 B k y}$ embryos display a forebrain defect very similar to, but even more severe than, cbs/ $c b s$ mutants of the same age. The reduced expression and lack of mutation in the Ift 88 mRNA in the cbs mutant indicates a novel hypomorphic allele that allows the embryos to live long enough to uncover major defects in dorsal telencephalic development, in contrast to the full knockout, which dies already at 10.5 d.p.c (Murcia et al., 2000). In contrast to other Ift88 mutations (Murcia et al., 2000; Haycraft et al., 2001, 2005, 2007; Kramer-Zucker et al., 2005; Banizs et al., 2005), ultrastructurally normal primary cilia are still to be found projecting into the telencephalic ventricle of $c b s / c b s$ mutants. We hypothesize that the observed levels of Ift 88 protein in the cbs mutant (25\% of wild-type levels) are sufficient to generate primary cilia, as demonstrated by TEM and scanning EM in the forebrain (Fig. 1) and elsewhere (supplemental Fig. 5, available at www.jneurosci.org as supplemental material), but are not sufficient to maintain the levels of signal transduction/protein processing necessary for proper development, as demonstrated by a reduction in Gli3 processing (Fig. $8 D$ ), as has been reported for a number of IFT mutants (Haycraft et al., 2005; Huangfu and Anderson, 2005; Liu et al., 2005; May et al., 2005; Tran et al., 2008). Another observation suggests that functional cilia are formed in early development in $c b s / c b s$ mutants. In knock-out mutations in IFT genes (Murcia et al., 2000; Liu et al., 2005), situs inversus has been correlated with a loss of cilia at the embryonic node (Murcia et al., 2000; Huangfu et al., 2003; Houde et al., 2006). Although $c b s / c b s$ mutants never displayed situs inversus, compound heterozygotes of the $c b s$ and Ift 88 knock-out alleles, in which the protein levels of Ift 88 are expected to be reduced further than in $c b s / c b s$ mutants, did display situs inversus. This suggests that the level of Ift 88 protein in the $c b s / c b s$ mutant lies just above the limit for ciliary formation, but is not high enough to allow for normal signal transduction. The levels of Ift 88 protein are also reduced in the Ift 88 hypomorph $T g 737^{\text {orp }}$, and a postnatal examination of brain ventricles in this mutant indicated that cilia were indeed present, but they were sparser, shorter, and showed altered morphology (Banizs et al., 2005). It could be expected that primary cilia in the $c b s / c b s$ mutants would also show morphological defects over time, but the early lethality of the embryos prevents this issue from being addressed.

The following observations allow us to conclude that the reduction of Ift 88 protein levels seen in the $c b s / c b s$ mutants results primarily in a defect in ciliary function. First, a large number of studies have reported the Ift 88 protein to be exclusively localized to the base and tips of primary cilia, in a wide variety of tissues (Taulman et al., 2001; Pazour et al., 2002; Haycraft et al., 2005, 2007). Second, Ift88 is well documented to be involved in IFT (Rosenbaum and Witman, 2002). Third, the alterations seen in Gli3 processing (Fig. 8E, F) have also been seen in a number of mutants in IFT proteins that are also known to be localized to primary cilia (Haycraft et al., 2005; Huangfu and Anderson, 2005; Liu et al., 2005; May et al., 2005; Tran et al., 2008). Fourth, two other IFT proteins have been reported to show defects in forebrain development. Null mutations in Dnchc2, a gene encoding the retrograde IFT motor, demonstrate a breakdown in the pallial-subpallial boundary (May et al., 2005), while a null mutant in Thm1, a novel protein that localizes to cilia and regulates retrograde IFT, demonstrates exencephaly and heterotopia-like structures in the cerebral cortex (Herron et al., 2002; Tran et al., 2008). In comparison with Ift 88 deletion mutants and other IFT mutants (Huangfu et al., 2003; May et al., 2005; Houde et al., 2006; Tran et al., 2008), cbs is therefore a novel mutation in that it shows no morphological ciliary defect but does indicate a reduction in ciliary function, through its altered processing of Gli3.

The $c b s$ phenotype bears strong resemblance to that of the Gli3 deletion mutant $X t^{J}$. Both mutants show abnormal development of the dorsomedial telencephalon and of the boundaries separating the dorsal telencephalon from the ventral telencephalon (Tole et al., 2000; Kuschel et al., 2003) and from the diencephalon (Theil et al., 1999; Fotaki et al., 2006). Both mutants are also characterized by ectopic $W n t 7 b$ expression in cortical progenitors (Theil, 2005) and by formation of heterotopias with a rosette 

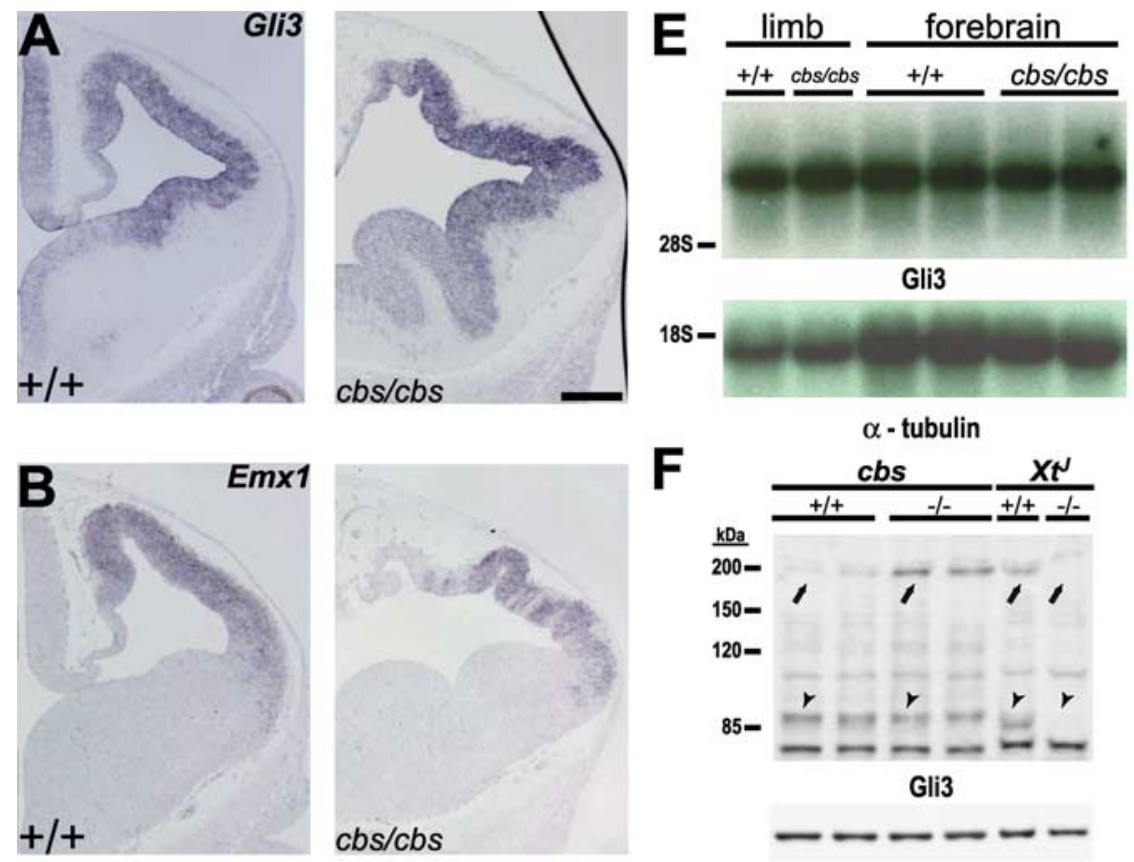

\section{$\mathbf{F}$}
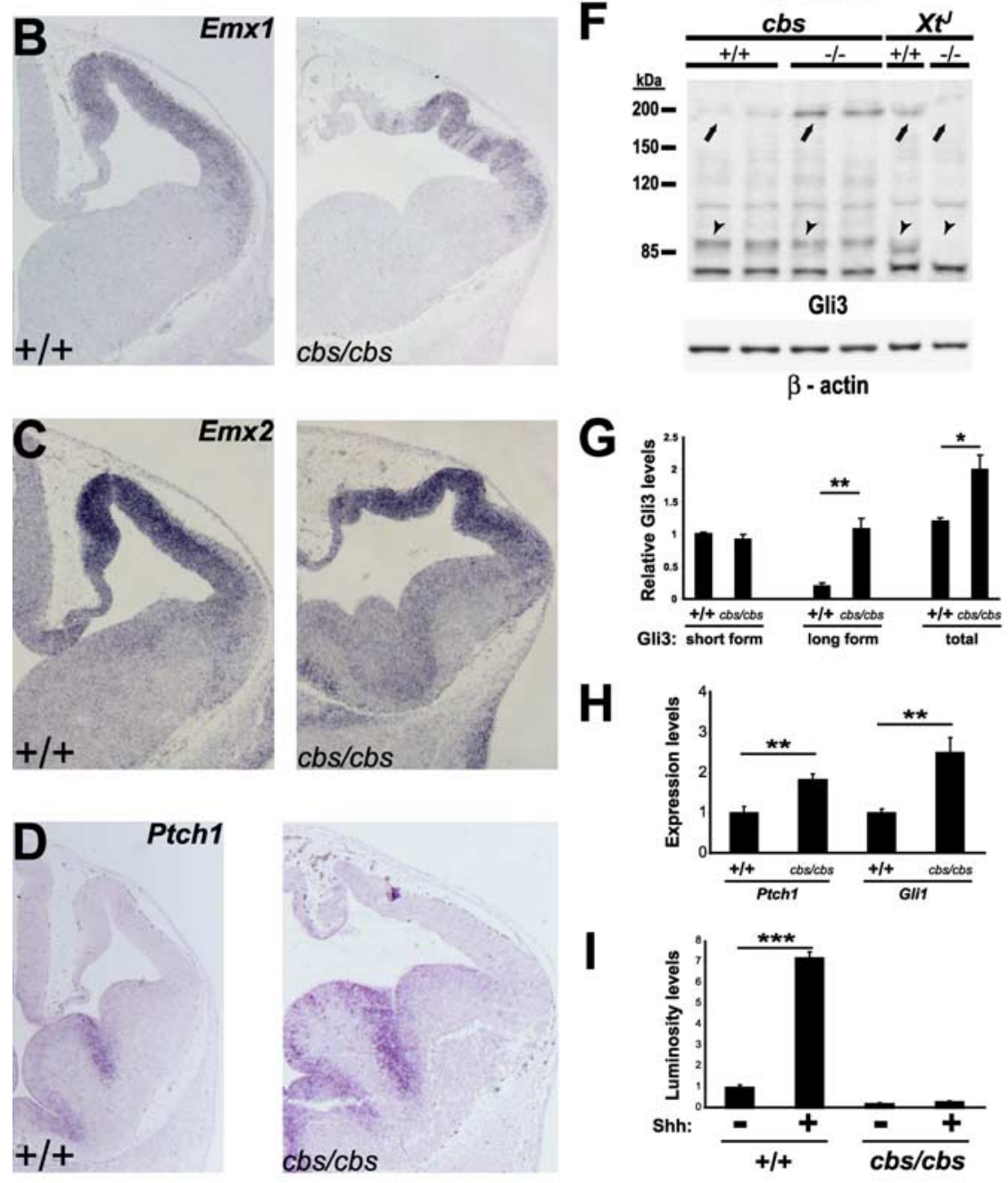

Figure 8. Targets of Shh signaling and Gli3 protein processing are disturbed in the forebrain of cbs mutants. $\boldsymbol{A}-\boldsymbol{D}$, In situ hybridization analysis of 12.5 wild-type and cbs/cbs embryos. For each coronal section, only one telencephalic half is shown, with dorsal to the top, lateral to the right. Scale bars: $300 \mu \mathrm{m}$. A, Gli3. B, Emx1.C, Emx2. D, Ptch1. E, Northern blots of whole RNA from forebrain of E12.5 wild-type and cbs/cbs embryos. Full-length Gli3 (top) and $\alpha$-tubulin cDNAs (bottom) were used as probes. Ribosomal RNA markers are to the left. $\boldsymbol{F}$, Western blots of protein from forebrain of E12.5 $c b s$ and $X t$ wild-type $(+/+)$ and homozygous mutant (-/-) embryos. An anti-N-terminal-Gli3 antibody (top) and an anti- $\beta$-actin antibody (bottom) were used. Specific bands corresponding to the $190 \mathrm{kDa}$ full-length Gli3 isoform (arrows) and the $80 \mathrm{kDa}$ proteolytically processed Gli3 isoform (arrowheads) are indicated. The specificity of the antibody was shown by examining homozygous Gli3 deletion mutants ( $\left.X_{t}^{\prime}\right)$, in which neither full-length nor processed Gli3 isoforms are detectable. Protein markers (kDa) are to the left. G, Quantitation of Gli3 Western blots seen in $(\boldsymbol{F})$, first indicating the amount of the $90 \mathrm{kDa}$ (short form) Gli3 isoform, setting levels in $+/+$ to 1.0. A quantitative comparison of the 190 $\mathrm{kDa}$ (long form) isoform shows $19.4 \%$ levels in + I+ embryos, compared with the short form. cbs/cbs mutants show a 5.6 -fold increase in the amount of the long form, compared with $+1+$ embryos, to levels greater than that of the short form in cbs/cbs embryos. The combined amount of short and long isoforms is also indicated (total). $\boldsymbol{H}$, Quantitative real time RT-PCR was performed upon mRNA extracted from E12.5 telencephalon. Reverse-transcribed CDNA was analyzed using TaqMan probes recognizing Ptch1 and Gli1. CDNA was normalized using probes for GAPDH. I, Luciferase assays using a Gli-responsive luciferase plasmid transiently transfected into fibroblasts prepared from wild-type and cbs/cbs embryos and allowed to reach confluency. Sonichedgehog $(\mathrm{Shh}, 1 \mu \mathrm{g} / \mathrm{ml})$ was added to the cultures for $12 \mathrm{~h}$ before lysis and analysis of luciferase levels. Relative luminosity levels relative to Shh-untreated wild-type cells are indicated. $\mathbf{G}-\mathbf{I}$, Mean values $\pm \operatorname{SEM}(n=4-8) .{ }^{* * *} p<0.001,{ }^{* *} p<0.01,{ }^{*} p<0.05$, Student's t test. structure (Theil et al., 1999; Fotaki et al., 2006). However, there are also important phenotypic differences. In contrast to $X t^{J}$ mutants, invagination and specification of dorsomedial structures (e.g., choroid plexus, cortical hem) occurs to some extent in the $c b s$ mutant, similar to Gli3 hypomorphic mutants (Kuschel et al., 2003; Friedrichs et al., 2008). Also, Emxl and Emx2 expression are retained in the neocortex of the $c b s$ mutant (Fig. $8 B, C$ ), indicative of a weaker Gli3 mutant phenotype. However, rosette formation starts earlier and is more extensive in the $c b s \mathrm{mu}-$ tant, suggesting a stronger phenotype compared with Gli3 mutants.

We hypothesize that the differences could lie in the nature of the $X t^{J}$ mutation, a genomic deletion of Gli3 in which both the full-length transcriptional activator as well as the processed transcriptional repressor forms are absent (Maynard et al., 2002). In contrast, $c b s / c b s$ mutants retain both of these protein isoforms, but the relative ratio of the two is shifted toward the activator form by a factor of five. Because of competition between the two isoforms, the effective concentration of Gli3 repressor could be further reduced, and Gli3 target genes which would be repressed in wild-type embryos would be activated in the $c b s / c b s$ forebrain inappropriately. However, the identity of these Gli3 genes targets in the cortex is not well understood. For example, although both $E m x 1$ and Emx2 are downregulated in the $X t^{J}$ mutant (Theil et al., 1999; Tole et al., 2000), Emx2 is not thought to be a direct transcriptional target of Gli3 (Theil et al., 2002). Therefore, the overproduction of the Gli activator in $c b s$ mutants is probably not directly linked to the expression of Emx2 in the $c b s$ mutant. In contrast, Wnt signaling has been shown to directly regulate the transcriptional activation of Emx2 (Theil et al., 2002). However, the upregulation that we observed of $W n t 7 b$ and $W n t 8 b$ was strongest in the caudal-most telencephalon, some distance away from the more rostral cortex where Emxl and Emx2 are expressed, although it is possible that diffusion of these growth factors could in fact induce Emx1/2 transcription. Regardless of their transcriptional control, the maintenance of their expression in the $c b s$ mutant, and the similar $c b s$ and $X t^{J}$ forebrain phenotypes, suggests that reduced Emx1/2 expression does not provide a major contribution to the $X t^{J}$ forebrain defects, consistent with the finding that a double Emx1/Emx2 knock-out did not reproduce many aspects of the $X t^{J}$ phenotype (Shinozaki et al., 2004). 
Surprisingly, we observed an upregulation of the Shhresponsive genes Ptch 1 and Gli1 in the $c b s / c b s$ ventral telencephalon. This stands in contrast to the reduction of Ptch 1 and Gli1 expression reported in the forelimb (Haycraft et al., 2005; Liu et al., 2005), hippocampus (Han et al., 2008), and cerebellum (Spassky et al., 2008) of several mouse mutants in IFT proteins. One possible explanation lies in the fact that cortical cilia are still present in the $c b s / c b s$ mutants, whereas they are not present in the brain (Han et al., 2008; Spassky et al., 2008) or the forelimb (Haycraft et al., 2005) of the mutants reported in three of the studies reported above. In IFT mutants in which cilia are lost, transcriptionally active Gli3 is not produced (Haycraft et al., 2005; Huangfu and Anderson, 2005; Liu et al., 2005), and it has been suggested that the presence of cilia is necessary for the production and release of a transcriptionally activating, full-length Gli3 isoform (Caspary et al., 2007). Interestingly, disruption of the gene encoding the ciliary protein Arl13b also resulted in an expansion of the Ptch1-expressing tissue in the spinal cord, reminiscent to the increase of Ptch1 expression that we see in the GEs of the $c b s / c b s$ mutant (Fig. $8 D$ ). We propose that the low levels of Ift 88 protein seen in the $c b s / c b s$ mutants, which clearly allow for the establishment of morphologically normal cilia, also allow for the production and accumulation of a transcriptional-activating, full-length Gli3 isoform, which is responsible for the observed upregulation of Ptch1 and Gli1. It is also possible that Gli2 may serve this function; not only has it been localized to cilia, but also its transcriptional-activating properties have been suggested to depend upon cilia (Haycraft et al., 2005). However, the deficiencies in IFT expected to arise from a $75 \%$ decrease in Ift 88 protein levels prohibit an acute transcriptional response to Shh treatment in vitro, as seen in the luciferase experiments on fibroblast cultures (Fig. 8I) and also reported for other IFT mutants (Ocbina and Anderson, 2008). In any case, altered Shh signaling is unlikely to contribute to the dorsal telencephalic phenotype found in the cbs/cbs mutants, as seen by comparison with the situation in the $X t^{I}$ mutant. In $X t^{I} / X t^{I}$ embryos, as in $c b s / c b s$ embryos, Shh signaling is not ectopically activated in the dorsal telencephalon (Theil et al., 1999). More importantly, the $X t^{J}$ mutant dorsal telencephalic phenotype is not rescued in $X t^{J} / S h h$ double mutant embryos (Rash and Grove, 2007).

It must also be kept in mind that other developmentally important proteins, such as Wnt signaling proteins (Corbit et al., 2008), have also been localized to cilia. Interestingly, we observed a clear upregulation of Axin2, a target of canonical Wnt signaling (Fig. 7), and an ectopic expression of $W n t 7 b$ and $W n t 8 b$ that may account for the Axin2 upregulation Although both Wnt7b and $W n t 8 b$ are seen to be upregulated in the $X t^{J}$ mutant, the upregulation in the caudal rosette-rich area of the $c b s$ mutant is more extensive than seen in the corresponding area of the $X t^{J}$ mutant brain, suggesting that it may be a direct consequence of ciliary misfunction. Clearly, further investigation is needed to dissect the relative contributions of Gli3 and Wnt family members to cilia-directed telencephalic development, and also to examine other roles that cilia may play at later stages in the differentiation of this complicated structure.

\section{References}

Agren M, Kogerman P, Kleman MI, Wessling M, Toftgård R (2004) Expression of the PTCH1 tumor suppressor gene is regulated by alternative promoters and a single functional Gli-binding site. Gene 330:101-114.

Banizs B, Pike MM, Millican CL, Ferguson WB, Komlosi P, Sheetz J, Bell PD,
Schwiebert EM, Yoder BK (2005) Dysfunctional cilia lead to altered ependyma and choroid plexus function, and result in the formation of hydrocephalus. Development 132:5329-5339.

Bisgrove BW, Yost HJ (2006) The roles of cilia in developmental disorders and disease. Development 133:4131-4143.

Brachmann I, Jakubick VC, Shakèd M, Unsicker K, Tucker KL (2007) A simple slice culture system for the imaging of nerve development in embryonic mouse. Dev Dyn 236:3514-3523.

Bulchand S, Grove EA, Porter FD, Tole S (2001) LIM-homeodomain gene Lhx2 regulates the formation of the cortical hem. Mech Dev 100:165-175.

Bulfone A, Puelles L, Porteus MH, Frohman MA, Martin GR, Rubenstein JL (1993) Spatially restricted expression of Dlx-1, Dlx-2 (Tes-1), Gbx-2, and Wnt-3 in the embryonic day 12.5 mouse forebrain defines potential transverse and longitudinal segmental boundaries. J Neurosci 13:3155-3172.

Caspary T, Larkins CE, Anderson KV (2007) The graded response to Sonic Hedgehog depends on cilia architecture. Dev Cell 12:767-778.

Chizhikov VV, Davenport J, Zhang Q, Shih EK, Cabello OA, Fuchs JL, Yoder BK, Millen KJ (2007) Cilia proteins control cerebellar morphogenesis by promoting expansion of the granule progenitor pool. J Neurosci 27:9780-9789.

Cohen E, Meininger V (1987) Ultrastructural analysis of primary cilium in the embryonic nervous tissue of mouse. Int J Dev Neurosci 5:43-51.

Corbit KC, Shyer AE, Dowdle WE, Gaulden J, Singla V, Chen MH, Chuang PT, Reiter JF (2008) Kif3a constrains beta-catenin-dependent Wnt signalling through dual ciliary and non-ciliary mechanisms. Nat Cell Biol 10:70-76.

Davis EE, Brueckner M, Katsanis N (2006) The emerging complexity of the vertebrate cilium: new functional roles for an ancient organelle. Dev Cell 11:9-19.

Dietrich WF, Miller JC, Steen RG, Merchant M, Damron D, Nahf R, Gross A, Joyce DC, Wessel M, Dredge RD, et al (1994) A genetic map of the mouse with 4,006 simple sequence length polymorphisms. Nat Genet 7:220-245.

Duan W, Cole T, Schreiber G (1989) Cloning and nucleotide sequencing of transthyretin (prealbumin) cDNA from rat choroid plexus and liver. Nucleic Acids Res 17:3979.

Fotaki V, Yu T, Zaki PA, Mason JO, Price DJ (2006) Abnormal positioning of diencephalic cell types in neocortical tissue in the dorsal telencephalon of mice lacking functional Gli3. J Neurosci 26:9282-9292.

Friedrichs M, Larralde O, Skutella T, Theil T (2008) Lamination of the cerebral cortex is disturbed in Gli3 mutant mice. Dev Biol 318:203-214

Fuchs JL, Schwark HD (2004) Neuronal primary cilia: a review. Cell Biol Int 28:111-118.

Gerdes JM, Liu Y, Zaghloul NA, Leitch CC, Lawson SS, Kato M, Beachy PA, Beales PL, DeMartino GN, Fisher S, Badano JL, Katsanis N (2007) Disruption of the basal body compromises proteasomal function and perturbs intracellular Wnt response. Nat Genet 39:1350-1360.

Goodrich LV, Johnson RL, Milenkovic L, McMahon JA, Scott MP (1996) Conservation of the hedgehog/patched signaling pathway from flies to mice: induction of a mouse patched gene by Hedgehog. Genes Dev 10:301-312.

Gorgas K (1984) Peroxisomes in sebaceous glands. V. Complex peroxisomes in the mouse preputial gland: serial sectioning and threedimensional reconstruction studies. Anat Embryol (Berl) 169:261-270.

Gradwohl G, Fode C, Guillemot F (1996) Restricted expression of a novel murine atonal-related bHLH protein in undifferentiated neural precursors. Dev Biol 180:227-241.

Grove EA, Tole S, Limon J, Yip L, Ragsdale CW (1998) The hem of the embryonic cerebral cortex is defined by the expression of multiple Wnt genes and is compromised in Gli3-deficient mice. Development 125:2315-2325.

Han YG, Spassky N, Romaguera-Ros M, Garcia-Verdugo JM, Aguilar A, Schneider-Maunoury S, Alvarez-Buylla A (2008) Hedgehog signaling and primary cilia are required for the formation of adult neural stem cells. Nat Neurosci 11:277-284.

Hanashima C, Shen L, Li SC, Lai E (2002) Brain factor-1 controls the proliferation and differentiation of neocortical progenitor cells through independent mechanisms. J Neurosci 22:6526-6536.

Hatini V, Huh SO, Herzlinger D, Soares VC, Lai E (1996) Essential role of stromal mesenchyme in kidney morphogenesis revealed by targeted dis- 
ruption of Winged Helix transcription factor BF-2. Genes Dev 10:1467-1478.

Haycraft CJ, Swoboda P, Taulman PD, Thomas JH, Yoder BK (2001) The C. elegans homolog of the murine cystic kidney disease gene $\mathrm{Tg} 737$ functions in a ciliogenic pathway and is disrupted in osm-5 mutant worms. Development 128:1493-1505.

Haycraft CJ, Banizs B, Aydin-Son Y, Zhang Q, Michaud EJ, Yoder BK (2005) Gli2 and Gli3 localize to cilia and require the intraflagellar transport protein polaris for processing and function. PLoS Genet 1:e53.

Haycraft CJ, Zhang Q, Song B, Jackson WS, Detloff PJ, Serra R, Yoder BK (2007) Intraflagellar transport is essential for endochondral bone formation. Development 134:307-316.

Herron BJ, Lu W, Rao C, Liu S, Peters H, Bronson RT, Justice MJ, McDonald JD, Beier DR (2002) Efficient generation and mapping of recessive developmental mutations using ENU mutagenesis. Nat Genet 30:185-189.

Houde C, Dickinson RJ, Houtzager VM, Cullum R, Montpetit R, Metzler M, Simpson EM, Roy S, Hayden MR, Hoodless PA, Nicholson DW (2006) Hippi is essential for node cilia assembly and Sonic hedgehog signaling. Dev Biol 300:523-533.

Huangfu D, Anderson KV (2005) Cilia and Hedgehog responsiveness in the mouse. Proc Natl Acad Sci U S A 102:11325-11330.

Huangfu D, Liu A, Rakeman AS, Murcia NS, Niswander L, Anderson KV (2003) Hedgehog signalling in the mouse requires intraflagellar transport proteins. Nature $426: 83-87$.

Hui CC, Joyner AL (1993) A mouse model of greig cephalopolysyndactyly syndrome: the extra-toes J mutation contains an intragenic deletion of the Gli3 gene. Nat Genet 3:241-246.

Huntzicker EG, Estay IS, Zhen H, Lokteva LA, Jackson PK, Oro AE (2006) Dual degradation signals control Gli protein stability and tumor formation. Genes Dev 20:276-281.

Jho EH, Zhang T, Domon C, Joo CK, Freund JN, Costantini F (2002) Wnt/ beta-catenin/Tcf signaling induces the transcription of Axin2, a negative regulator of the signaling pathway. Mol Cell Biol 22:1172-1183.

Johnson DR (1967) Extra-toes: a new mutant gene causing multiple abnormalities in the mouse. J Embryol Exp Morphol 17:543-581.

Kramer-Zucker AG, Olale F, Haycraft CJ, Yoder BK, Schier AF, Drummond IA (2005) Cilia-driven fluid flow in the zebrafish pronephros, brain and Kupffer's vesicle is required for normal organogenesis. Development 132:1907-1921.

Kuschel S, Rüther U, Theil T (2003) A disrupted balance between Bmp/Wnt and Fgf signaling underlies the ventralization of the Gli3 mutant telencephalon. Dev Biol 260:484-495.

Lee J, Platt KA, Censullo P, Ruiz i Altaba A (1997) Gli1 is a target of Sonic hedgehog that induces ventral neural tube development. Development 124:2537-2552.

Lemischka IR, Farmer S, Racaniello VR, Sharp PA (1981) Nucleotide sequence and evolution of a mammalian alpha-tubulin messenger RNA. J Mol Biol 151:101-120.

Liu A, Wang B, Niswander LA (2005) Mouse intraflagellar transport proteins regulate both the activator and repressor functions of Gli transcription factors. Development 132:3103-3111.

Lustig B, Jerchow B, Sachs M, Weiler S, Pietsch T, Karsten U, van de Wetering M, Clevers H, Schlag PM, Birchmeier W, Behrens J (2002) Negative feedback loop of Wnt signaling through upregulation of conductin/axin2 in colorectal and liver tumors. Mol Cell Biol 22:1184-1193.

Mangale VS, Hirokawa KE, Satyaki PR, Gokulchandran N, Chikbire S, Subramanian L, Shetty AS, Martynoga B, Paul J, Mai MV, Li Y, Flanagan LA, Tole S, Monuki ES (2008) Lhx2 selector activity specifies cortical identity and suppresses hippocampal organizer fate. Science 319:304-309.

Marigo V, Davey RA, Zuo Y, Cunningham JM, Tabin CJ (1996) Biochemical evidence that patched is the Hedgehog receptor. Nature 384:176-179.

May SR, Ashique AM, Karlen M, Wang B, Shen Y, Zarbalis K, Reiter J, Ericson J, Peterson AS (2005) Loss of the retrograde motor for IFT disrupts localization of Smo to cilia and prevents the expression of both activator and repressor functions of Gli. Dev Biol 287:378-389.

Maynard TM, Jain MD, Balmer CW, LaMantia AS (2002) High-resolution mapping of the Gli3 mutation extra-toes reveals a $51.5-\mathrm{kb}$ deletion. Mamm Genome 13:58-61.

Medina L, Legaz I, Gonzalez G, De Castro F, Rubenstein JL, Puelles L
(2004) Expression of Dbxl, Neurogenin 2, Semaphorin 5A, Cadherin 8, and Emxl distinguish ventral and laterial pallial histogenetic divisions in the developing mouse claustroamygdaloid complex. J Comp Neurol 474:504-523.

Meyer G, Perez-Garcia CG, Abraham H, Caput D (2002) Expression of p73 and Reelin in the developing human cortex. J Neurosci 22:4973-4986.

Monuki ES, Porter FD, Walsh CA (2001) Patterning of the dorsal telencephalon and cerebral cortex by a roof plate-Lhx2 pathway. Neuron 32:591-604.

Moody SA, Quigg MS, Frankfurter A (1989) Development of the peripheral trigeminal system in the chick revealed by an isotype-specific anti-betatubulin monoclonal antibody. J Comp Neurol 279:567-580.

Murcia NS, Richards WG, Yoder BK, Mucenski ML, Dunlap JR, Woychik RP (2000) The Oak Ridge Polycystic Kidney (orpk) disease gene is required for left-right axis determination. Development 127:2347-2355.

Nagele RG, Lee HY (1979) Ultrastructural changes in cells associated with interkinetic nuclear migration in the developing chick neuroepithelium. J Exp Zool 210:89-106.

Ocbina PJ, Anderson KV (2008) Intraflagellar transport, cilia, and mammalian Hedgehog signaling: analysis in mouse embryonic fibroblasts. Dev Dyn 237:2030-2038.

Pazour GJ, Baker SA, Deane JA, Cole DG, Dickert BL, Rosenbaum JL, Witman GB, Besharse JC (2002) The intraflagellar transport protein, IFT88, is essential for vertebrate photoreceptor assembly and maintenance. J Cell Biol 157:103-113.

Platt KA, Michaud J, Joyner AL (1997) Expression of the mouse Gli and Ptc genes is adjacent to embryonic sources of hedgehog signals suggesting a conservation of pathways between flies and mice. Mech Dev 62:121-135.

Porter FD, Drago J, Xu Y, Cheema SS, Wassif C, Huang SP, Lee E, Grinberg A, Massalas JS, Bodine D, Alt F, Westphal H (1997) Lhx2, a LIM homeobox gene, is required for eye, forebrain, and definitive erythrocyte development. Development 124:2935-2944.

Rash BG, Grove EA (2007) Patterning the dorsal telencephalon: a role for sonic hedgehog? J Neurosci 27:11595-11603.

Rosenbaum JL, Witman GB (2002) Intraflagellar transport. Nat Rev Mol Cell Biol 3:813-825.

Sasaki H, Hui C, Nakafuku M, Kondoh H (1997) A binding site for Gli proteins is essential for HNF-3beta floor plate enhancer activity in transgenics and can respond to Shh in vitro. Development 124:1313-1322.

Scholey JM, Anderson KV (2006) Intraflagellar transport and cilium-based signaling. Cell 125:439-442.

Shinozaki K, Yoshida M, Nakamura M, Aizawa S, Suda Y (2004) Emxl and Emx2 cooperate in initial phase of archipallium development. Mech Dev 121:475-489.

Spassky N, Han YG, Aguilar A, Strehl L, Besse L, Laclef C, Ros MR, GarciaVerdugo JM, Alvarez-Buylla A (2008) Primary cilia are required for cerebellar development and Shh-dependent expansion of progenitor pool. Dev Biol 317:246-259.

Takiguchi-Hayashi K, Sekiguchi M, Ashigaki S, Takamatsu M, Hasegawa H, Suzuki-Migishima R, Yokoyama M, Nakanishi S, Tanabe Y (2004) Generation of reelin-positive marginal zone cells from the caudomedial wall of telencephalic vesicles. J Neurosci 24:2286-2295.

Tao W, Lai E (1992) Telencephalon-restricted expression of BF-1, a new member of the HNF-3/fork head gene family, in the developing rat brain. Neuron 8:957-966.

Taulman PD, Haycraft CJ, Balkovetz DF, Yoder BK (2001) Polaris, a protein involved in left-right axis patterning, localizes to basal bodies and cilia. Mol Biol Cell 12:589-599.

Theil T (2005) Gli3 is required for the specification and differentiation of preplate neurons. Dev Biol 286:559-571.

Theil T, Alvarez-Bolado G, Walter A, Rüther U (1999) Gli3 is required for Emx gene expression during dorsal telencephalon development. Development 126:3561-3571.

Theil T, Aydin S, Koch S, Grotewold L, Rüther U (2002) Wnt and Bmp signalling cooperatively regulate graded Emx2 expression in the dorsal telencephalon. Development 129:3045-3054.

Tole S, Ragsdale CW, Grove EA (2000) Dorsoventral patterning of the telencephalon is disrupted in the mouse mutant extra-toes $(J)$. Dev Biol 217:254-265. 
Tran PV, Haycraft CJ, Besschetnova TY, Turbe-Doan A, Stottmann RW, Herron BJ, Chesebro AL, Qiu H, Scherz PJ, Shah JV, Yoder BK, Beier DR (2008) THM1 negatively modulates mouse sonic hedgehog signal transduction and affects retrograde intraflagellar transport in cilia. Nat Genet 40:403-410.

Tucker KL, Beard C, Dausmann J, Jackson-Grusby L, Laird PW, Lei H, Li E, Jaenisch R (1996) Germ-line passage is required for establishment of methylation and expression patterns of imprinted but not of nonimprinted genes. Genes Dev 10:1008-1020.

Tucker KL, Wang Y, Dausman J, Jaenisch R (1997) A transgenic mouse strain expressing four drug-selectable marker genes. Nucleic Acids Res 25:3745-3746.

Tucker KL, Meyer M, Barde YA (2001) Neurotrophins are required for nerve growth during development. Nat Neurosci 4:29-37.
Walther C, Gruss P (1991) Pax-6, a murine paired box gene, is expressed in the developing CNS. Development 113:1435-1449.

Wang B, Fallon JF, Beachy PA (2000) Hedgehog-regulated processing of Gli3 produces an anterior/posterior repressor gradient in the developing vertebrate limb. Cell 100:423-434.

Yamashita T, Tucker KL, Barde YA (1999) Neurotrophin binding to the p75 receptor modulates Rho activity and axonal outgrowth. Neuron 24:585-593.

Yun K, Potter S, Rubenstein JL (2001) Gsh2 and Pax6 play complementary roles in dorsoventral patterning of the mammalian telencephalon. Development 128:193-205.

Zhang Q, Murcia NS, Chittenden LR, Richards WG, Michaud EJ, Woychik RP, Yoder BK (2003) Loss of the Tg737 protein results in skeletal patterning defects. Dev Dyn 227:78-90. 